\title{
The Gauss-Bonnet theorem for noncommutative two tori with a general conformal structure
}

\author{
Farzad Fathizadeh and Masoud Khalkhali
}

\begin{abstract}
In this paper we give a proof of the Gauss-Bonnet theorem of Connes and Tretkoff for noncommutative two tori $\mathbb{T}_{\theta}^{2}$ equipped with an arbitrary translation invariant complex structure. More precisely, we show that for any complex number $\tau$ in the upper half plane, representing the conformal class of a metric on $\mathbb{T}_{\theta}^{2}$, and a Weyl factor given by a positive invertible element $k \in C^{\infty}\left(\mathbb{T}_{\theta}^{2}\right)$, the value at the origin, $\zeta(0)$, of the spectral zeta function of the Laplacian $\triangle^{\prime}$ attached to $\left(\mathbb{T}_{\theta}^{2}, \tau, k\right)$ is independent of $\tau$ and $k$.
\end{abstract}

Mathematics Subject Classification (2010). 58B34.

Keywords. Noncommutative torus, Gauss-Bonnet theorem, spectral zeta function, Connes' pseudodifferential calculus.

\section{Introduction}

In this paper we extend the Gauss-Bonnet theorem of Connes and Tretkoff [8] (cf. also [4] for a preliminary version) for the noncommutative two torus $\mathbb{T}_{\theta}^{2}$, to noncommutative two tori equipped with an arbitrary translation invariant complex structure. In fact this more general result was already indicated in [8]. More precisely, we show that for any complex number $\tau$ in the upper half plane, representing the conformal class of a metric on $\mathbb{T}_{\theta}^{2}$, and a Weyl factor given by a positive invertible element $k \in C^{\infty}\left(\mathbb{\nabla}_{\theta}^{2}\right)$, the value at the origin, $\zeta(0)$, of the spectral zeta function of the Laplacian $\Delta^{\prime}$ attached to $\left(\mathbb{T}_{\theta}^{2}, \tau, k\right)$ is independent of $\tau$ and $k$. The main ideas in the proof are those employed and invented in [8] to treat the case $\tau=\sqrt{-1}$, namely a pseudodifferential calculus for the canonical dynamical system associated to the noncommutative torus, and the use of the asymptotic expansion of the heat kernel in computing the zeta values. This, however, by itself is not enough and, similar to [8], one needs an extra and intricate argument to express $\zeta(0)$ in terms of the modular operator defined by the Weyl factor. As a first step, the calculation of the asymptotic expansion of the heat operator for arbitrary values of the conformal class $\tau$ is quite involved and must be performed by a computer. We found it impossible to carry this step without the use of symbolic calculations. Finally we should mention that, as is explained in [8], there is a close relationship between the subject of this paper and 
scale invariance in spectral action [2], [3] on the one hand, and non-unimodular (or twisted) spectral triples [7] on the other hand.

It is a great pleasure to thank Alain Connes for motivating and enlightening discussions on the topic of this paper. We would also like to thank German Combariza Gonzales, Farideh Fazayeli, and Eric Schost for their generous help with symbolic calculations with GAP.

\section{Preliminaries}

Let $\Sigma$ be a closed, oriented, 2-dimensional smooth manifold equipped with a Riemannian metric $g$. Recall that the spectral zeta function $\zeta(s)$ associated to the Laplacian $\triangle_{g}=d^{*} d$ of $(\Sigma, g)$ is given by the following summation over the non-zero eigenvalues $\lambda_{j}$ of $\triangle_{g}$ :

$$
\zeta(s)=\sum \lambda_{j}^{-s}, \quad \operatorname{Re}(s)>1 .
$$

The zeta function has a meromorphic continuation to $\mathbb{C}$ with a unique (simple) pole at $s=1$. In particular $\zeta(0)$ is defined and we have

$$
\zeta(0)+\operatorname{Card}\left\{j \mid \lambda_{j}=0\right\}=\frac{1}{12 \pi} \int_{\Sigma} R=\frac{1}{6} \chi(\Sigma),
$$

where $R$ is the scalar curvature and $\chi(\Sigma)$ is the Euler-Poincaré characteristic. Thus $\zeta(0)$ is a topological invariant, and, in particular, it remains invariant under the conformal perturbation $g \rightarrow e^{f} g$ of the metric [9], [10].

Next, we recall the definition of the spectral zeta function for the Laplacian on the noncommutative two torus from [8]. Let $\theta$ be an irrational number. Recall that the irrational rotation $C^{*}$-algebra $A_{\theta}$ is, by definition, the universal unital $C^{*}$-algebra generated by two unitaries $U, V$ satisfying

$$
V U=e^{2 \pi i \theta} U V .
$$

One usually thinks of $A_{\theta}$ as the algebra of continuous functions on the noncommutative 2-torus $\mathbb{T}_{\theta}$. There is a continuous action of $\mathbb{T}^{2}, \mathbb{T}=\mathbb{R} / 2 \pi \mathbb{Z}$, on $A_{\theta}$ by $C^{*}$-algebra automorphisms $\left\{\alpha_{s}\right\}, s \in \mathbb{R}^{2}$, defined by

$$
\alpha_{s}\left(U^{m} V^{n}\right)=e^{i s .(m, n)} U^{m} V^{n} .
$$

The space of smooth elements for this action, that is those elements $a \in A_{\theta}$ for which the map $s \mapsto \alpha_{s}(a)$ is $C^{\infty}$ will be denoted by $A_{\theta}^{\infty}$. It is a dense subalgebra of $A_{\theta}$ which can be alternatively described as the algebra of elements in $A_{\theta}$ whose (noncommutative) Fourier expansion has rapidly decreasing coefficients:

$A_{\theta}^{\infty}=\left\{\sum_{m, n \in \mathbb{Z}} a_{m, n} U^{m} V^{n} \mid\left(|m|^{k}|n|^{q}\left|a_{m, n}\right|\right)_{m, n}\right.$ is bounded for any positive $\left.k, q\right\}$. 
There is a unique normalized trace $\tau_{0}$ on $A_{\theta}$ whose restriction on smooth elements is given by

$$
\tau_{0}\left(\sum_{m, n \in \mathbb{Z}} a_{m, n} U^{m} V^{n}\right)=a_{0,0} .
$$

The infinitesimal generators of the above action of $\mathbb{T}^{2}$ on $A_{\theta}$ are the derivations $\delta_{1}, \delta_{2}: A_{\theta}^{\infty} \rightarrow A_{\theta}^{\infty}$ defined by

$$
\delta_{1}(U)=U, \delta_{1}(V)=0, \quad \delta_{2}(U)=0, \delta_{2}(V)=V .
$$

In fact, $\delta_{1}, \delta_{2}$ are analogues of the differential operators $\frac{1}{i} \partial / \partial x, \frac{1}{i} \partial / \partial y$ acting on the smooth functions on the ordinary two torus. We have $\delta_{j}\left(a^{*}\right)=-\delta_{j}(a)^{*}$ for $j=1,2$ and all $a \in A_{\theta}^{\infty}$. Moreover, since $\tau_{0} \circ \delta_{j}=0$, for $j=1,2$, we have the analogue of the integration by parts formula:

$$
\tau_{0}\left(a \delta_{j}(b)\right)=-\tau_{0}\left(\delta_{j}(a) b\right) \text { for all } a, b \in A_{\theta}^{\infty} .
$$

We define an inner product on $A_{\theta}$ by

$$
\langle a, b\rangle=\tau_{0}\left(b^{*} a\right), \quad a, b \in A_{\theta},
$$

and complete $A_{\theta}$ with respect to this inner product to obtain a Hilbert space denoted by $\mathscr{H}_{0}$. The derivations $\delta_{1}, \delta_{2}$, as unbounded operators on $\mathscr{H}_{0}$, are formally selfadjoint and have unique extensions to selfadjoint operators.

We introduce a complex structure associated with a complex number $\tau=\tau_{1}+i \tau_{2}$, $\tau_{2}>0$, by defining

$$
\partial=\delta_{1}+\tau \delta_{2}, \quad \partial^{*}=\delta_{1}+\bar{\tau} \delta_{2} .
$$

To the conformal structure defined by $\tau$ corresponds a positive Hochschild two cocycle on $A_{\theta}^{\infty}$ given by (cf. [6])

$$
\psi(a, b, c)=-\tau_{0}\left(a \partial b \partial^{*} c\right)
$$

We note that $\partial$ is an unbounded operator on $\mathscr{H}_{0}$ and $\partial^{*}$ is its formal adjoint. The analogue of the space of $(1,0)$-forms on the ordinary two torus is defined to be the Hilbert space completion of the space of finite sums $\sum a \partial b, a, b \in A_{\theta}^{\infty}$, with respect to the inner product defined above, and it is denoted by $\mathscr{H}^{(1,0)}$. Now we view $\partial$ as an unbounded operator from $\mathscr{H}_{0}$ to $\mathscr{H}^{(1,0)}$ and define the Laplacian $\triangle$ on $A_{\theta}^{\infty}$ by

$$
\triangle:=\partial^{*} \partial=\delta_{1}^{2}+2 \tau_{1} \delta_{1} \delta_{2}+|\tau|^{2} \delta_{2}^{2}
$$

In order to investigate the analogue of the Gauss-Bonnet theorem for the noncommutative torus [8], we vary inside the conformal class of the metric by choosing a smooth selfadjoint element $h=h^{*} \in A_{\theta}^{\infty}$ and define a linear functional $\varphi$ on $A_{\theta}$ by

$$
\varphi(a)=\tau_{0}\left(a e^{-h}\right), \quad a \in A_{\theta}
$$


In fact, $\varphi$ is a positive linear functional which is not a trace, however, it is a twisted trace and satisfies the KMS condition at $\beta=1$ for the 1-parameter group $\left\{\sigma_{t}\right\}, t \in \mathbb{R}$, of inner automorphisms $\sigma_{t}=\Delta^{-i t}$, where the modular operator for $\varphi$ is given by (cf. [8])

$$
\Delta(x)=e^{-h} x e^{h} .
$$

Moreover, the 1-parameter group of automorphisms $\sigma_{t}$ is generated by the derivation $-\log \Delta$, where

$$
\log \Delta(x)=[-h, x], \quad x \in A_{\theta}^{\infty} .
$$

We define an inner product $\langle,\rangle_{\varphi}$ on $A_{\theta}$ by

$$
\langle a, b\rangle_{\varphi}=\varphi\left(b^{*} a\right), \quad a, b \in A_{\theta} .
$$

The Hilbert space obtained from completing $A_{\theta}$ with respect to this inner product will be denoted by $\mathscr{H}_{\varphi}$. Now let $\partial_{\varphi}$ be the same operator as $\partial$, but viewed as an unbounded operator from $\mathscr{H}_{\varphi}$ to $\mathscr{H}^{(1,0)}$, and define the modified Laplacian $\triangle^{\prime}$ by

$$
\triangle^{\prime}:=\partial_{\varphi}^{*} \partial_{\varphi}
$$

Obviously, $\triangle^{\prime}$ is a positive unbounded operator acting in $\mathscr{H}_{\varphi}$.

Lemma 2.1. Let $k=e^{h / 2} \in A_{\theta}$ act in the Hilbert space $\mathscr{H}_{0}$ by left multiplication. Then the operator $\triangle^{\prime}$ acting in the Hilbert space $\mathcal{H}_{\varphi}$ is anti-unitarily equivalent to the operator $k \Delta k$ acting in $\mathscr{H}_{0}$.

Proof. The proof is very similar to that of Connes and Tretkoff given in Lemma 2.1 in [8]. The right multiplication by $k$ extends to a unitary map $W: \mathscr{H}_{0} \rightarrow \mathscr{H}_{\varphi}$ since

$$
\langle W(a), W(b)\rangle_{\varphi}=\tau_{0}\left((b k)^{*}(a k) k^{-2}\right)=\tau_{0}\left(k b^{*} a k k^{-2}\right)=\tau_{0}\left(b^{*} a\right)=\langle a, b\rangle .
$$

Obviously $\partial_{\varphi} \circ W=\partial_{\varphi} \circ R_{k}$, both acting in $\mathscr{H}_{0}$. Therefore $\left(\partial_{\varphi} \circ W\right)^{*}\left(\partial_{\varphi} \circ W\right)=$ $\left(\partial_{\varphi} \circ R_{k}\right)^{*}\left(\partial_{\varphi} \circ R_{k}\right)$ which, since $R_{k}^{*}=R_{k}$, yields

$$
W^{*} \triangle^{\prime} W=R_{k} \triangle R_{k} .
$$

Therefore the operators $\Delta^{\prime}$ and $R_{k} \triangle R_{k}$, acting in $\mathscr{H}_{\varphi}$ and $\mathscr{H}_{0}$ respectively, are unitarily equivalent. Now let $J$ be the involution on $\mathscr{H}_{0}$ given by $J(a)=a^{*}$ for all $a \in A_{\theta}$ which is anti-unitary. Since the operator $J$ commutes with $\triangle=\delta_{1}^{2}+$ $2 \tau_{1} \delta_{1} \delta_{2}+|\tau|^{2} \delta_{2}^{2}$ and satisfies $J R_{k} J=k$, we have

$$
J R_{k} \triangle R_{k} J=J R_{k} J J \triangle J J R_{k} J=k \triangle k .
$$

Therefore the operators $\triangle^{\prime}$ and $k \Delta k$ are anti-unitarily equivalent.

Remark 2.1. To have an explicit formula for the adjoint of $\partial_{\varphi}$, one can easily check that

$$
\partial_{\varphi}^{*}=R_{k^{2}} \partial^{*} .
$$




\section{Pseudodifferential operators and $\zeta(0)$}

As we saw in Section 2, the operator $\Delta^{\prime} \sim k \Delta k$ is a positive unbounded operator. Similar to the classical case, using the formula (1), one can associate a spectral zeta function to this operator. The main result of this paper states that the value at the origin of the zeta function of the operator $\Delta^{\prime} \sim k \Delta k$ is independent of the choice of the invertible positive element $k \in A_{\theta}^{\infty}$ and the conformal class of the metric defined by $\tau \in \mathbb{C} \backslash \mathbb{R}$. Accordingly, this section is intended to briefly discuss the notion of a pseudodifferential operator associated to the dynamical system $\left(A_{\theta}^{\infty}, \alpha_{s}\right)$ [1], [5], and to explain how this will help to compute the value at the origin of the spectral zeta function of an elliptic operator. For a more detailed discussion, we refer the reader to [8], [9].

For a non-negative integer $n$, the space of differential operators on $A_{\theta}^{\infty}$ of order at most $n$ is defined to be the vector space of operators of the form

$$
\sum_{j_{1}+j_{2} \leq n} a_{j_{1}, j_{2}} \delta_{1}^{j_{1}} \delta_{2}^{j_{2}}, \quad j_{1}, j_{2} \geq 0, a_{j_{1}, j_{2}} \in A_{\theta}^{\infty} .
$$

The notion of a differential operator on $A_{\theta}^{\infty}$ can be generalized to the notion of a pseudodifferential operator using operator valued symbols. In the sequel, we shall use the notation $\partial_{1}=\frac{\partial}{\partial \xi_{1}}, \partial_{2}=\frac{\partial}{\partial \xi_{2}}$.

Definition 3.1. For an integer $n$, a smooth map $\rho: \mathbb{R}^{2} \rightarrow A_{\theta}^{\infty}$ is said to be a symbol of order $n$ if for all non-negative integers $i_{1}, i_{2}, j_{1}, j_{2}$,

$$
\left\|\delta_{1}^{i_{1}} \delta_{2}^{i_{2}} \partial_{1}^{j_{1}} \partial_{2}^{j_{2}} \rho(\xi)\right\| \leq c(1+|\xi|)^{n-j_{1}-j_{2}}
$$

where $c$ is a constant, and if there exists a smooth map $k: \mathbb{R}^{2} \rightarrow A_{\theta}^{\infty}$ such that

$$
\lim _{\lambda \rightarrow \infty} \lambda^{-n} \rho\left(\lambda \xi_{1}, \lambda \xi_{2}\right)=k\left(\xi_{1}, \xi_{2}\right)
$$

The space of symbols of order $n$ is denoted by $S_{n}$.

To a symbol $\rho$ of order $n$, one can associate an operator on $A_{\theta}^{\infty}$, denoted by $P_{\rho}$, given by

$$
P_{\rho}(a)=(2 \pi)^{-2} \iint e^{-i s \cdot \xi} \rho(\xi) \alpha_{s}(a) d s d \xi
$$

The operator $P_{\rho}$ is said to be a pseudodifferential operator of order $n$. For example, the differential operator $\sum_{j_{1}+j_{2} \leq n} a_{j_{1}, j_{2}} \delta_{1}^{j_{1}} \delta_{2}^{j_{2}}$ is associated with the symbol $\sum_{j_{1}+j_{2} \leq n} a_{j_{1}, j_{2}} \xi_{1}^{j_{1}} \xi_{2}^{j_{2}}$ via the above formula.

Definition 3.2. Two symbols $\rho, \rho^{\prime} \in S_{k}$ are said to be equivalent if and only if $\rho-\rho^{\prime}$ is in $S_{n}$ for all integers $n$. The equivalence of the symbols will be denoted by $\rho \sim \rho^{\prime}$. 
The following lemma shows that the space of pseudodifferential operators is an algebra and one can find the symbol of the product of pseudodifferential operators up to the above equivalence. Also, the adjoint of a pseudodifferential operator, with respect to the inner product defined on $\mathscr{H}_{0}$ in Section 2, is a pseudodifferential operator with the symbol given in the following proposition up to the equivalence (cf. [8]).

Proposition 3.1. Let $P$ and $Q$ be pseudodifferential operators with the symbols $\rho$ and $\rho^{\prime}$, respectively. Then the adjoint $P^{*}$ and the product $P Q$ are pseudodifferential operators with symbols

$$
\begin{aligned}
\sigma\left(P^{*}\right) & \sim \sum_{\ell_{1}, \ell_{2} \geq 0} \frac{1}{\ell_{1} ! \ell_{2} !} \partial_{1}^{\ell_{1}} \partial_{2}^{\ell_{2}} \delta_{1}^{\ell_{1}} \delta_{2}^{\ell_{2}}(\rho(\xi))^{*}, \\
\sigma(P Q) & \sim \sum_{\ell_{1}, \ell_{2} \geq 0} \frac{1}{\ell_{1} ! \ell_{2} !} \partial_{1}^{\ell_{1}} \partial_{2}^{\ell_{2}}(\rho(\xi)) \delta_{1}^{\ell_{1}} \delta_{2}^{\ell_{2}}\left(\rho^{\prime}(\xi)\right) .
\end{aligned}
$$

Definition 3.3. Let $\rho$ be a symbol of order $n$. It is said to be elliptic if $\rho(\xi)$ is invertible for $\xi \neq 0$ and if there exists a constant $c$ such that

$$
\left\|\rho(\xi)^{-1}\right\| \leq c(1+|\xi|)^{-n}
$$

for sufficiently large $|\xi|$.

The operator $\Delta=\delta_{1}^{2}+2 \tau_{1} \delta_{1} \delta_{2}+|\tau|^{2} \delta_{2}^{2}$ defined in Section 2 is an example of an elliptic operator.

In order to study the value at the origin of the $\zeta$ function of the operator $\Delta^{\prime} \sim k \Delta k$, one can use the Mellin transform to write

$$
\zeta(s)=\operatorname{Tr}\left(\triangle^{\prime-s}\right)=\frac{1}{\Gamma(s)} \int_{0}^{\infty} \operatorname{Trace}^{+}\left(e^{-t \Delta^{\prime}}\right) t^{s-1} d t,
$$

where $\operatorname{Trace}^{+}\left(e^{-t \Delta^{\prime}}\right)=\operatorname{Trace}\left(e^{-t \Delta^{\prime}}\right)-\operatorname{Dim} \operatorname{Ker}\left(\Delta^{\prime}\right)$.

Using the Cauchy integral formula, one has

$$
e^{-t \Delta^{\prime}}=\frac{1}{2 \pi i} \int_{C} e^{-t \lambda}\left(\triangle^{\prime}-\lambda\right)^{-1} d \lambda,
$$

where $C$ is a curve in the complex plane that goes around the non-negative real axis in the anti-clockwise direction without touching it. Appealing to this formula and using similar arguments to those of [9], one can derive the following asymptotic expansion:

$$
\operatorname{Trace}\left(e^{-t \Delta^{\prime}}\right) \sim t^{-1} \sum_{n=0}^{\infty} B_{2 n}\left(\triangle^{\prime}\right) t^{n}, \quad t \rightarrow 0^{+} .
$$

Considering the fact that $\Gamma$ has a simple pole at $s=0$ with residue 1 and using the above asymptotic expansion, one can see that

$$
\zeta(0)+1=B_{2}\left(\triangle^{\prime}\right) .
$$


In order to find the latter, similar to the formula in [9], one can approximate the inverse of the operator $\left(\triangle^{\prime}-\lambda\right)$ by a pseudodifferential operator $B_{\lambda}$ whose symbol has an expansion of the form

$$
b_{0}(\xi, \lambda)+b_{1}(\xi, \lambda)+b_{2}(\xi, \lambda)+\cdots,
$$

where $b_{j}(\xi, \lambda)$ is a symbol of order $-2-j$ and

$$
\sigma\left(B_{\lambda}\left(\triangle^{\prime}-\lambda\right)\right) \sim 1
$$

Then one can see that

$$
B_{2}\left(\triangle^{\prime}\right)=\frac{1}{2 \pi i} \iint_{C} e^{-\lambda} \tau_{0}\left(b_{2}(\xi, \lambda)\right) d \lambda d \xi
$$

To compute the latter, by a homogeneity argument (cf. [8]), one can set $\lambda=-1$ and multiply the answer by -1 . Therefore

$$
\zeta(0)+1=-\int \tau_{0}\left(b_{2}(\xi,-1)\right) d \xi .
$$

\section{The computation of $\zeta(0)$}

In order to compute the value at the origin of the zeta function of the operator $\triangle^{\prime} \sim$ $k \Delta k$ by the method explained in Section 3, first we find the symbol of this operator.

Lemma 4.1. The operator $k \Delta k$ has symbol $a_{2}(\xi)+a_{1}(\xi)+a_{0}(\xi)$, where

$$
\begin{aligned}
& a_{2}(\xi)=\xi_{1}^{2} k^{2}+|\tau|^{2} \xi_{2}^{2} k^{2}+2 \tau_{1} \xi_{1} \xi_{2} k^{2}, \\
& a_{1}(\xi)=2 \xi_{1} k \delta_{1}(k)+2|\tau|^{2} \xi_{2} k \delta_{2}(k)+2 \tau_{1} \xi_{1} k \delta_{2}(k)+2 \tau_{1} \xi_{2} k \delta_{1}(k), \\
& a_{0}(\xi)=k \delta_{1}^{2}(k)+|\tau|^{2} k \delta_{2}^{2}(k)+2 \tau_{1} k \delta_{1} \delta_{2}(k) .
\end{aligned}
$$

Proof. The above expressions are derived by applying the formula for the symbol of product of pseudodifferential operators explained in Proposition 3.1 to the symbol of left multiplication by $k$, which is $k$, and to the symbol of $\triangle$, which is $\xi_{1}^{2}+2 \tau_{1} \xi_{1} \xi_{2}+$ $|\tau|^{2} \xi_{2}^{2}$.

Similar to the calculation of the inverse of the symbol of $\triangle^{\prime}-\lambda$ in [9], now we can find the symbols $b_{j}(\xi)$ for $j=0,1,2, \ldots$ such that each $b_{j}$ is of order $-2-j$ and

$$
\left(b_{0}+b_{1}+b_{2}+\cdots\right) \sigma\left(\triangle^{\prime}+1\right)=\left(b_{0}+b_{1}+b_{2}+\cdots\right)\left(\left(a_{2}+1\right)+a_{1}+a_{0}\right) \sim 1 \text {. }
$$


Note that, without any loss in the generality for computing $\zeta(0)$, we have set $\lambda=-1$. Let $a_{2}^{\prime}=a_{2}+1, a_{1}^{\prime}=a_{1}, a_{0}^{\prime}=a_{0}$. In fact, 1 is treated as a symbol of order 2 . Then the above equation yields

$$
\sum_{\substack{j, \ell_{1}, \ell_{2} \geq 0 \\ k=0,1,2}} \frac{1}{\ell_{1} ! \ell_{2} !} \partial_{1}^{\ell_{1}} \partial_{2}^{\ell_{2}}\left(b_{j}\right) \delta_{1}^{\ell_{1}} \delta_{2}^{\ell_{2}}\left(a_{k}^{\prime}\right) \sim 1 .
$$

Since $\partial_{1}^{\ell_{1}} \partial_{2}^{\ell_{2}}\left(b_{j}\right) \delta_{1}^{\ell_{1}} \delta_{2}^{\ell_{2}}\left(a_{k}^{\prime}\right)$ is a symbol of order $-2-j-\left(\ell_{1}+\ell_{2}\right)+k$, one can decompose the above series into homogeneous terms of order $-n$ for $n \geq 0$ and write the above equation as

$$
\sum_{n \geq 0} \sum_{\substack{j, \ell_{1}, \ell_{2} \geq 0, k=0,1,2 \\ 2+j+\ell_{1}+\ell_{2}-k=n}} \frac{1}{\ell_{1} ! \ell_{2} !} \partial_{1}^{\ell_{1}} \partial_{2}^{\ell_{2}}\left(b_{j}\right) \delta_{1}^{\ell_{1}} \delta_{2}^{\ell_{2}}\left(a_{k}^{\prime}\right) \sim 1 .
$$

Now by comparing the symbols of order $n=0$ on both sides, we have $b_{0} a_{2}^{\prime}=1$, therefore

$$
b_{0}=a_{2}^{\prime-1}=\left(a_{2}+1\right)^{-1}=\left(\xi_{1}^{2} k^{2}+|\tau|^{2} \xi_{2}^{2} k^{2}+2 \tau_{1} \xi_{1} \xi_{2} k^{2}+1\right)^{-1} .
$$

Inductively, one gets

$$
b_{n} a_{2}^{\prime}=-\sum_{\substack{2+j+\ell_{1}+\ell_{2}-k=n \\ 0 \leq j<n, 0 \leq k \leq 2}} \frac{1}{\ell_{1} ! \ell_{2} !} \partial_{1}^{\ell_{1}} \partial_{2}^{\ell_{2}}\left(b_{j}\right) \delta_{1}^{\ell_{1}} \delta_{2}^{\ell_{2}}\left(a_{k}^{\prime}\right) .
$$

If $\mathrm{k}=2$ then $\ell_{1}+\ell_{2}>0$, therefore $\delta_{1}^{\ell_{1}} \delta_{2}^{\ell_{2}}\left(a_{k}^{\prime}\right)=\delta_{1}^{\ell_{1}} \delta_{2}^{\ell_{2}}\left(a_{k}\right)$ in the latter. Hence, for $n>0$,

$$
b_{n}=-\sum_{\substack{2+j+\ell_{1}+\ell_{2}-k=n \\ 0 \leq j<n, 0 \leq k \leq 2}} \frac{1}{\ell_{1} ! \ell_{2} !} \partial_{1}^{\ell_{1}} \partial_{2}^{\ell_{2}}\left(b_{j}\right) \delta_{1}^{\ell_{1}} \delta_{2}^{\ell_{2}}\left(a_{k}\right) b_{0} .
$$

In particular, we have

$$
\begin{aligned}
b_{1}= & -\left(b_{0} a_{1} b_{0}+\partial_{1}\left(b_{0}\right) \delta_{1}\left(a_{2}\right) b_{0}+\partial_{2}\left(b_{0}\right) \delta_{2}\left(a_{2}\right) b_{0}\right), \\
b_{2}= & -\left(b_{0} a_{0} b_{0}+b_{1} a_{1} b_{0}+\partial_{1}\left(b_{0}\right) \delta_{1}\left(a_{1}\right) b_{0}+\partial_{2}\left(b_{0}\right) \delta_{2}\left(a_{1}\right) b_{0}\right. \\
& +\partial_{1}\left(b_{1}\right) \delta_{1}\left(a_{2}\right) b_{0}+\partial_{2}\left(b_{1}\right) \delta_{2}\left(a_{2}\right) b_{0}+(1 / 2) \partial_{11}\left(b_{0}\right) \delta_{1}^{2}\left(a_{2}\right) b_{0} \\
& \left.+(1 / 2) \partial_{22}\left(b_{0}\right) \delta_{2}^{2}\left(a_{2}\right) b_{0}+\partial_{12}\left(b_{0}\right) \delta_{1} \delta_{2}\left(a_{2}\right) b_{0}\right) .
\end{aligned}
$$

After computing these terms directly, one finds that $b_{2}$ is equal to a very long expression which will not be reproduced here since it would occupy eight full pages. Invoking the trace property of $\tau_{0}$, we can permute the factors of each term cyclically and obtain the following terms for computing $\zeta(0)$ : 
Gauss-Bonnet theorem for noncommutative 2-tori with a general conformal structure 465

$$
\begin{aligned}
& -b_{0}^{2} k \delta_{1}^{2}(k)-2 \tau_{1} b_{0}^{2} k \delta_{1} \delta_{2}(k)-|\tau|^{2} b_{0}^{2} k \delta_{2}^{2}(k) \\
& +\left(6 \xi_{1}^{2}+12 \tau_{1} \xi_{1} \xi_{2}+4 \tau_{1}^{2} \xi_{2}^{2}+2|\tau|^{2} \xi_{2}^{2}\right) b_{0}^{3} k^{3} \delta_{1}^{2}(k) \\
& +\left(6 \xi_{1}^{2}+12 \tau_{1} \xi_{1} \xi_{2}+4 \tau_{1}^{2} \xi_{2}^{2}+2|\tau|^{2} \xi_{2}^{2}\right) b_{0}^{3} k^{2} \delta_{1}(k)^{2} \\
& +\left(2 \xi_{1}^{2}+4 \tau_{1} \xi_{1} \xi_{2}+2|\tau|^{2} \xi_{2}^{2}\right) b_{0}^{2} k^{2} \delta_{1}(k) b_{0} \delta_{1}(k) \\
& +\left(6 \xi_{1}^{2}+12 \tau_{1} \xi_{1} \xi_{2}+4 \tau_{1}^{2} \xi_{2}^{2}+2|\tau|^{2} \xi_{2}^{2}\right) b_{0}^{2} k \delta_{1}(k) b_{0} k \delta_{1}(k) \\
& +\left(12 \tau_{1} \xi_{1}^{2}+16 \tau_{1}^{2} \xi_{1} \xi_{2}+8|\tau|^{2} \xi_{1} \xi_{2}+12 \tau_{1}|\tau|^{2} \xi_{2}^{2}\right) b_{0}^{3} k^{3} \delta_{1} \delta_{2}(k) \\
& +\left(6 \tau_{1} \xi_{1}^{2}+8 \tau_{1}^{2} \xi_{1} \xi_{2}+4|\tau|^{2} \xi_{1} \xi_{2}+6 \tau_{1}|\tau|^{2} \xi_{2}^{2}\right) b_{0}^{3} k^{2} \delta_{1}(k) \delta_{2}(k) \\
& +\left(6 \tau_{1} \xi_{1}^{2}+8 \tau_{1}^{2} \xi_{1} \xi_{2}+4|\tau|^{2} \xi_{1} \xi_{2}+6 \tau_{1}|\tau|^{2} \xi_{2}^{2}\right) b_{0}^{3} k^{2} \delta_{2}(k) \delta_{1}(k) \\
& +\left(2 \tau_{1} \xi_{1}^{2}+4 \tau_{1}^{2} \xi_{1} \xi_{2}+2 \tau_{1}|\tau|^{2} \xi_{2}^{2}\right) b_{0}^{2} k^{2} \delta_{1}(k) b_{0} \delta_{2}(k) \\
& +\left(2 \tau_{1} \xi_{1}^{2}+4 \tau_{1}^{2} \xi_{1} \xi_{2}+2 \tau_{1}|\tau|^{2} \xi_{2}^{2}\right) b_{0}^{2} k^{2} \delta_{2}(k) b_{0} \delta_{1}(k) \\
& +\left(6 \tau_{1} \xi_{1}^{2}+8 \tau_{1}^{2} \xi_{1} \xi_{2}+4|\tau|^{2} \xi_{1} \xi_{2}+6 \tau_{1}|\tau|^{2} \xi_{2}^{2}\right) b_{0}^{2} k \delta_{1}(k) b_{0} k \delta_{2}(k) \\
& +\left(6 \tau_{1} \xi_{1}^{2}+8 \tau_{1}^{2} \xi_{1} \xi_{2}+4|\tau|^{2} \xi_{1} \xi_{2}+6 \tau_{1}|\tau|^{2} \xi_{2}^{2}\right) b_{0}^{2} k \delta_{2}(k) b_{0} k \delta_{1}(k) \\
& +\left(4 \tau_{1}^{2} \xi_{1}^{2}+2|\tau|^{2} \xi_{1}^{2}+12 \tau_{1}|\tau|^{2} \xi_{1} \xi_{2}+6|\tau|^{4} \xi_{2}^{2}\right) b_{0}^{3} k^{3} \delta_{2}^{2}(k) \\
& +\left(4 \tau_{1}^{2} \xi_{1}^{2}+2|\tau|^{2} \xi_{1}^{2}+12 \tau_{1}|\tau|^{2} \xi_{1} \xi_{2}+6|\tau|^{4} \xi_{2}^{2}\right) b_{0}^{3} k^{2} \delta_{2}(k)^{2} \\
& +\left(4 \tau_{1}^{2} \xi_{1}^{2}+2|\tau|^{2} \xi_{1}^{2}+12 \tau_{1}|\tau|^{2} \xi_{1} \xi_{2}+6|\tau|^{4} \xi_{2}^{2}\right) b_{0}^{2} k \delta_{2}(k) b_{0} k \delta_{2}(k) \\
& +\left(2|\tau|^{2} \xi_{1}^{2}+4 \tau_{1}|\tau|^{2} \xi_{1} \xi_{2}+2|\tau|^{4} \xi_{2}^{2}\right) b_{0}^{2} k^{2} \delta_{2}(k) b_{0} \delta_{2}(k) \\
& +\left(-8 \xi_{1}^{4}-32 \tau_{1} \xi_{1}^{3} \xi_{2}-40 \tau_{1}^{2} \xi_{1}^{2} \xi_{2}^{2}-8|\tau|^{2} \xi_{1}^{2} \xi_{2}^{2}\right. \\
& \left.-16 \tau_{1}^{3} \xi_{1} \xi_{2}^{3}-16 \tau_{1}|\tau|^{2} \xi_{1} \xi_{2}^{3}-8 \tau_{1}^{2}|\tau|^{2} \xi_{2}^{4}\right) b_{0}^{4} k^{5} \delta_{1}^{2}(k) \\
& +\left(-8 \xi_{1}^{4}-32 \tau_{1} \xi_{1}^{3} \xi_{2}-40 \tau_{1}^{2} \xi_{1}^{2} \xi_{2}^{2}-8|\tau|^{2} \xi_{1}^{2} \xi_{2}^{2}\right. \\
& \left.-16 \tau_{1}^{3} \xi_{1} \xi_{2}^{3}-16 \tau_{1}|\tau|^{2} \xi_{1} \xi_{2}^{3}-8 \tau_{1}^{2}|\tau|^{2} \xi_{2}^{4}\right) b_{0}^{4} k^{4} \delta_{1}(k)^{2} \\
& +\left(-10 \xi_{1}^{4}-40 \tau_{1} \xi_{1}^{3} \xi_{2}-48 \tau_{1}^{2} \xi_{1}^{2} \xi_{2}^{2}-12|\tau|^{2} \xi_{1}^{2} \xi_{2}^{2}-16 \tau_{1}^{3} \xi_{1} \xi_{2}^{3}\right. \\
& \left.-24 \tau_{1}|\tau|^{2} \xi_{1} \xi_{2}^{3}-8 \tau_{1}^{2}|\tau|^{2} \xi_{2}^{4}-2|\tau|^{4} \xi_{2}^{4}\right) b_{0}^{3} k^{4} \delta_{1}(k) b_{0} \delta_{1}(k) \\
& +\left(-20 \xi_{1}^{4}-80 \tau_{1} \xi_{1}^{3} \xi_{2}-96 \tau_{1}^{2} \xi_{1}^{2} \xi_{2}^{2}-24|\tau|^{2} \xi_{1}^{2} \xi_{2}^{2}-32 \tau_{1}^{3} \xi_{1} \xi_{2}^{3}\right. \\
& \left.-48 \tau_{1}|\tau|^{2} \xi_{1} \xi_{2}^{3}-16 \tau_{1}^{2}|\tau|^{2} \xi_{2}^{4}-4|\tau|^{4} \xi_{2}^{4}\right) b_{0}^{3} k^{3} \delta_{1}(k) b_{0} k \delta_{1}(k) \\
& +\left(-10 \xi_{1}^{4}-40 \tau_{1} \xi_{1}^{3} \xi_{2}-48 \tau_{1}^{2} \xi_{1}^{2} \xi_{2}^{2}-12|\tau|^{2} \xi_{1}^{2} \xi_{2}^{2}-16 \tau_{1}^{3} \xi_{1} \xi_{2}^{3}\right. \\
& \left.-24 \tau_{1}|\tau|^{2} \xi_{1} \xi_{2}^{3}-8 \tau_{1}^{2}|\tau|^{2} \xi_{2}^{4}-2|\tau|^{4} \xi_{2}^{4}\right) b_{0}^{3} k^{2} \delta_{1}(k) b_{0} k^{2} \delta_{1}(k) \\
& +\left(-4 \xi_{1}^{4}-16 \tau_{1} \xi_{1}^{3} \xi_{2}-20 \tau_{1}^{2} \xi_{1}^{2} \xi_{2}^{2}-4|\tau|^{2} \xi_{1}^{2} \xi_{2}^{2}-8 \tau_{1}^{3} \xi_{1} \xi_{2}^{3}\right. \\
& \left.-8 \tau_{1}|\tau|^{2} \xi_{1} \xi_{2}^{3}-4 \tau_{1}^{2}|\tau|^{2} \xi_{2}^{4}\right) b_{0}^{2} k^{2} \delta_{1}(k) b_{0}^{2} k^{2} \delta_{1}(k) \\
& +\left(-4 \xi_{1}^{4}-16 \tau_{1} \xi_{1}^{3} \xi_{2}-20 \tau_{1}^{2} \xi_{1}^{2} \xi_{2}^{2}-4|\tau|^{2} \xi_{1}^{2} \xi_{2}^{2}-8 \tau_{1}^{3} \xi_{1} \xi_{2}^{3}\right. \\
& \left.-8 \tau_{1}|\tau|^{2} \xi_{1} \xi_{2}^{3}-4 \tau_{1}^{2}|\tau|^{2} \xi_{2}^{4}\right) b_{0}^{2} k \delta_{1}(k) b_{0}^{2} k^{3} \delta_{1}(k) \\
& +\left(-16 \tau_{1} \xi_{1}^{4}-48 \tau_{1}^{2} \xi_{1}^{3} \xi_{2}-16|\tau|^{2} \xi_{1}^{3} \xi_{2}-32 \tau_{1}^{3} \xi_{1}^{2} \xi_{2}^{2}-64 \tau_{1}|\tau|^{2} \xi_{1}^{2} \xi_{2}^{2}\right. \\
& \left.-48 \tau_{1}^{2}|\tau|^{2} \xi_{1} \xi_{2}^{3}-16|\tau|^{4} \xi_{1} \xi_{2}^{3}-16 \tau_{1}|\tau|^{4} \xi_{2}^{4}\right) b_{0}^{4} k^{5} \delta_{1} \delta_{2}(k)
\end{aligned}
$$




$$
\begin{aligned}
& +\left(-8 \tau_{1} \xi_{1}^{4}-24 \tau_{1}^{2} \xi_{1}^{3} \xi_{2}-8|\tau|^{2} \xi_{1}^{3} \xi_{2}-16 \tau_{1}^{3} \xi_{1}^{2} \xi_{2}^{2}-32 \tau_{1}|\tau|^{2} \xi_{1}^{2} \xi_{2}^{2}\right. \\
& \left.-24 \tau_{1}^{2}|\tau|^{2} \xi_{1} \xi_{2}^{3}-8|\tau|^{4} \xi_{1} \xi_{2}^{3}-8 \tau_{1}|\tau|^{4} \xi_{2}^{4}\right) b_{0}^{4} k^{4} \delta_{1}(k) \delta_{2}(k) \\
& +\left(-8 \tau_{1} \xi_{1}^{4}-24 \tau_{1}^{2} \xi_{1}^{3} \xi_{2}-8|\tau|^{2} \xi_{1}^{3} \xi_{2}-16 \tau_{1}^{3} \xi_{1}^{2} \xi_{2}^{2}-32 \tau_{1}|\tau|^{2} \xi_{1}^{2} \xi_{2}^{2}\right. \\
& \left.-24 \tau_{1}^{2}|\tau|^{2} \xi_{1} \xi_{2}^{3}-8|\tau|^{4} \xi_{1} \xi_{2}^{3}-8 \tau_{1}|\tau|^{4} \xi_{2}^{4}\right) b_{0}^{4} k^{4} \delta_{2}(k) \delta_{1}(k) \\
& +\left(-10 \tau_{1} \xi_{1}^{4}-32 \tau_{1}^{2} \xi_{1}^{3} \xi_{2}-8|\tau|^{2} \xi_{1}^{3} \xi_{2}-24 \tau_{1}^{3} \xi_{1}^{2} \xi_{2}^{2}-36 \tau_{1}|\tau|^{2} \xi_{1}^{2} \xi_{2}^{2}\right. \\
& \left.-32 \tau_{1}^{2}|\tau|^{2} \xi_{1} \xi_{2}^{3}-8|\tau|^{4} \xi_{1} \xi_{2}^{3}-10 \tau_{1}|\tau|^{4} \xi_{2}^{4}\right) b_{0}^{3} k^{4} \delta_{1}(k) b_{0} \delta_{2}(k) \\
& +\left(-10 \tau_{1} \xi_{1}^{4}-32 \tau_{1}^{2} \xi_{1}^{3} \xi_{2}-8|\tau|^{2} \xi_{1}^{3} \xi_{2}-24 \tau_{1}^{3} \xi_{1}^{2} \xi_{2}^{2}-36 \tau_{1}|\tau|^{2} \xi_{1}^{2} \xi_{2}^{2}\right. \\
& \left.-32 \tau_{1}^{2}|\tau|^{2} \xi_{1} \xi_{2}^{3}-8|\tau|^{4} \xi_{1} \xi_{2}^{3}-10 \tau_{1}|\tau|^{4} \xi_{2}^{4}\right) b_{0}^{3} k^{4} \delta_{2}(k) b_{0} \delta_{1}(k) \\
& +\left(-20 \tau_{1} \xi_{1}^{4}-64 \tau_{1}^{2} \xi_{1}^{3} \xi_{2}-16|\tau|^{2} \xi_{1}^{3} \xi_{2}-48 \tau_{1}^{3} \xi_{1}^{2} \xi_{2}^{2}-72 \tau_{1}|\tau|^{2} \xi_{1}^{2} \xi_{2}^{2}\right. \\
& \left.-64 \tau_{1}^{2}|\tau|^{2} \xi_{1} \xi_{2}^{3}-16|\tau|^{4} \xi_{1} \xi_{2}^{3}-20 \tau_{1}|\tau|^{4} \xi_{2}^{4}\right) b_{0}^{3} k^{3} \delta_{1}(k) b_{0} k \delta_{2}(k) \\
& +\left(-20 \tau_{1} \xi_{1}^{4}-64 \tau_{1}^{2} \xi_{1}^{3} \xi_{2}-16|\tau|^{2} \xi_{1}^{3} \xi_{2}-48 \tau_{1}^{3} \xi_{1}^{2} \xi_{2}^{2}-72 \tau_{1}|\tau|^{2} \xi_{1}^{2} \xi_{2}^{2}\right. \\
& \left.-64 \tau_{1}^{2}|\tau|^{2} \xi_{1} \xi_{2}^{3}-16|\tau|^{4} \xi_{1} \xi_{2}^{3}-20 \tau_{1}|\tau|^{4} \xi_{2}^{4}\right) b_{0}^{3} k^{3} \delta_{2}(k) b_{0} k \delta_{1}(k) \\
& +\left(-10 \tau_{1} \xi_{1}^{4}-32 \tau_{1}^{2} \xi_{1}^{3} \xi_{2}-8|\tau|^{2} \xi_{1}^{3} \xi_{2}-24 \tau_{1}^{3} \xi_{1}^{2} \xi_{2}^{2}-36 \tau_{1}|\tau|^{2} \xi_{1}^{2} \xi_{2}^{2}\right. \\
& \left.-32 \tau_{1}^{2}|\tau|^{2} \xi_{1} \xi_{2}^{3}-8|\tau|^{4} \xi_{1} \xi_{2}^{3}-10 \tau_{1}|\tau|^{4} \xi_{2}^{4}\right) b_{0}^{3} k^{2} \delta_{1}(k) b_{0} k^{2} \delta_{2}(k) \\
& +\left(-10 \tau_{1} \xi_{1}^{4}-32 \tau_{1}^{2} \xi_{1}^{3} \xi_{2}-8|\tau|^{2} \xi_{1}^{3} \xi_{2}-24 \tau_{1}^{3} \xi_{1}^{2} \xi_{2}^{2}-36 \tau_{1}|\tau|^{2} \xi_{1}^{2} \xi_{2}^{2}\right. \\
& \left.-32 \tau_{1}^{2}|\tau|^{2} \xi_{1} \xi_{2}^{3}-8|\tau|^{4} \xi_{1} \xi_{2}^{3}-10 \tau_{1}|\tau|^{4} \xi_{2}^{4}\right) b_{0}^{3} k^{2} \delta_{2}(k) b_{0} k^{2} \delta_{1}(k) \\
& +\left(-8 \tau_{1} \xi_{1}^{4}-24 \tau_{1}^{2} \xi_{1}^{3} \xi_{2}-8|\tau|^{2} \xi_{1}^{3} \xi_{2}-16 \tau_{1}^{3} \xi_{1}^{2} \xi_{2}^{2}-32 \tau_{1}|\tau|^{2} \xi_{1}^{2} \xi_{2}^{2}\right. \\
& \left.-24 \tau_{1}^{2}|\tau|^{2} \xi_{1} \xi_{2}^{3}-8|\tau|^{4} \xi_{1} \xi_{2}^{3}-8 \tau_{1}|\tau|^{4} \xi_{2}^{4}\right) b_{0}^{2} k^{2} \delta_{1}(k) b_{0}^{2} k^{2} \delta_{2}(k) \\
& +\left(-4 \tau_{1} \xi_{1}^{4}-12 \tau_{1}^{2} \xi_{1}^{3} \xi_{2}-4|\tau|^{2} \xi_{1}^{3} \xi_{2}-8 \tau_{1}^{3} \xi_{1}^{2} \xi_{2}^{2}-16 \tau_{1}|\tau|^{2} \xi_{1}^{2} \xi_{2}^{2}\right. \\
& \left.-12 \tau_{1}^{2}|\tau|^{2} \xi_{1} \xi_{2}^{3}+-4|\tau|^{4} \xi_{1} \xi_{2}^{3}-4 \tau_{1}|\tau|^{4} \xi_{2}^{4}\right) b_{0}^{2} k \delta_{1}(k) b_{0}^{2} k^{3} \delta_{2}(k) \\
& +\left(-4 \tau_{1} \xi_{1}^{4}-12 \tau_{1}^{2} \xi_{1}^{3} \xi_{2}-4|\tau|^{2} \xi_{1}^{3} \xi_{2}-8 \tau_{1}^{3} \xi_{1}^{2} \xi_{2}^{2}-16 \tau_{1}|\tau|^{2} \xi_{1}^{2} \xi_{2}^{2}\right. \\
& \left.-12 \tau_{1}^{2}|\tau|^{2} \xi_{1} \xi_{2}^{3}-4|\tau|^{4} \xi_{1} \xi_{2}^{3}-4 \tau_{1}|\tau|^{4} \xi_{2}^{4}\right) b_{0}^{2} k \delta_{2}(k) b_{0}^{2} k^{3} \delta_{1}(k) \\
& +\left(-8 \tau_{1}^{2} \xi_{1}^{4}-16 \tau_{1}^{3} \xi_{1}^{3} \xi_{2}-16 \tau_{1}|\tau|^{2} \xi_{1}^{3} \xi_{2}-40 \tau_{1}^{2}|\tau|^{2} \xi_{1}^{2} \xi_{2}^{2}\right. \\
& \left.-8|\tau|^{4} \xi_{1}^{2} \xi_{2}^{2}-32 \tau_{1}|\tau|^{4} \xi_{1} \xi_{2}^{3}-8|\tau|^{6} \xi_{2}^{4}\right) b_{0}^{4} k^{5} \delta_{2}^{2}(k) \\
& +\left(-8 \tau_{1}^{2} \xi_{1}^{4}-16 \tau_{1}^{3} \xi_{1}^{3} \xi_{2}-16 \tau_{1}|\tau|^{2} \xi_{1}^{3} \xi_{2}-40 \tau_{1}^{2}|\tau|^{2} \xi_{1}^{2} \xi_{2}^{2}\right. \\
& \left.-8|\tau|^{4} \xi_{1}^{2} \xi_{2}^{2}-32 \tau_{1}|\tau|^{4} \xi_{1} \xi_{2}^{3}-8|\tau|^{6} \xi_{2}^{4}\right) b_{0}^{4} k^{4} \delta_{2}(k)^{2} \\
& +\left(-8 \tau_{1}^{2} \xi_{1}^{4}-2|\tau|^{2} \xi_{1}^{4}-16 \tau_{1}^{3} \xi_{1}^{3} \xi_{2}-24 \tau_{1}|\tau|^{2} \xi_{1}^{3} \xi_{2}-48 \tau_{1}^{2}|\tau|^{2} \xi_{1}^{2} \xi_{2}^{2}\right. \\
& \left.-12|\tau|^{4} \xi_{1}^{2} \xi_{2}^{2}-40 \tau_{1}|\tau|^{4} \xi_{1} \xi_{2}^{3}-10|\tau|^{6} \xi_{2}^{4}\right) b_{0}^{3} k^{4} \delta_{2}(k) b_{0} \delta_{2}(k) \\
& +\left(-16 \tau_{1}^{2} \xi_{1}^{4}-4|\tau|^{2} \xi_{1}^{4}-32 \tau_{1}^{3} \xi_{1}^{3} \xi_{2}-48 \tau_{1}|\tau|^{2} \xi_{1}^{3} \xi_{2}-96 \tau_{1}^{2}|\tau|^{2} \xi_{1}^{2} \xi_{2}^{2}\right. \\
& \left.-24|\tau|^{4} \xi_{1}^{2} \xi_{2}^{2}-80 \tau_{1}|\tau|^{4} \xi_{1} \xi_{2}^{3}-20|\tau|^{6} \xi_{2}^{4}\right) b_{0}^{3} k^{3} \delta_{2}(k) b_{0} k \delta_{2}(k) \\
& +\left(-8 \tau_{1}^{2} \xi_{1}^{4}-2|\tau|^{2} \xi_{1}^{4}-16 \tau_{1}^{3} \xi_{1}^{3} \xi_{2}-24 \tau_{1}|\tau|^{2} \xi_{1}^{3} \xi_{2}-48 \tau_{1}^{2}|\tau|^{2} \xi_{1}^{2} \xi_{2}^{2}\right. \\
& \left.-12|\tau|^{4} \xi_{1}^{2} \xi_{2}^{2}-40 \tau_{1}|\tau|^{4} \xi_{1} \xi_{2}^{3}-10|\tau|^{6} \xi_{2}^{4}\right) b_{0}^{3} k^{2} \delta_{2}(k) b_{0} k^{2} \delta_{2}(k)
\end{aligned}
$$


Gauss-Bonnet theorem for noncommutative 2-tori with a general conformal structure 467

$$
\begin{aligned}
& +\left(-4 \tau_{1}^{2} \xi_{1}^{4}-8 \tau_{1}^{3} \xi_{1}^{3} \xi_{2}-8 \tau_{1}|\tau|^{2} \xi_{1}^{3} \xi_{2}-20 \tau_{1}^{2}|\tau|^{2} \xi_{1}^{2} \xi_{2}^{2}-4|\tau|^{4} \xi_{1}^{2} \xi_{2}^{2}\right. \\
& \left.-16 \tau_{1}|\tau|^{4} \xi_{1} \xi_{2}^{3}-4|\tau|^{6} \xi_{2}^{4}\right) b_{0}^{2} k^{2} \delta_{2}(k) b_{0}^{2} k^{2} \delta_{2}(k) \\
& +\left(-4 \tau_{1}^{2} \xi_{1}^{4}-8 \tau_{1}^{3} \xi_{1}^{3} \xi_{2}-8 \tau_{1}|\tau|^{2} \xi_{1}^{3} \xi_{2}-20 \tau_{1}^{2}|\tau|^{2} \xi_{1}^{2} \xi_{2}^{2}-4|\tau|^{4} \xi_{1}^{2} \xi_{2}^{2}\right. \\
& \left.-16 \tau_{1}|\tau|^{4} \xi_{1} \xi_{2}^{3}-4|\tau|^{6} \xi_{2}^{4}\right) b_{0}^{2} k \delta_{2}(k) b_{0}^{2} k^{3} \delta_{2}(k) \\
& +\left(8 \xi_{1}^{6}+48 \tau_{1} \xi_{1}^{5} \xi_{2}+104 \tau_{1}^{2} \xi_{1}^{4} \xi_{2}^{2}+16|\tau|^{2} \xi_{1}^{4} \xi_{2}^{2}+96 \tau_{1}^{3} \xi_{1}^{3} \xi_{2}^{3}\right. \\
& +64 \tau_{1}|\tau|^{2} \xi_{1}^{3} \xi_{2}^{3}+32 \tau_{1}^{4} \xi_{1}^{2} \xi_{2}^{4}+80 \tau_{1}^{2}|\tau|^{2} \xi_{1}^{2} \xi_{2}^{4}+8|\tau|^{4} \xi_{1}^{2} \xi_{2}^{4} \\
& \left.+32 \tau_{1}^{3}|\tau|^{2} \xi_{1} \xi_{2}^{5}+16 \tau_{1}|\tau|^{4} \xi_{1} \xi_{2}^{5}+8 \tau_{1}^{2}|\tau|^{4} \xi_{2}^{6}\right) b_{0}^{4} k^{6} \delta_{1}(k) b_{0} \delta_{1}(k) \\
& +\left(16 \xi_{1}^{6}+96 \tau_{1} \xi_{1}^{5} \xi_{2}+(208) \tau_{1}^{2} \xi_{1}^{4} \xi_{2}^{2}+32|\tau|^{2} \xi_{1}^{4} \xi_{2}^{2}+192 \tau_{1}^{3} \xi_{1}^{3} \xi_{2}^{3}\right. \\
& +128 \tau_{1}|\tau|^{2} \xi_{1}^{3} \xi_{2}^{3}+64 \tau_{1}^{4} \xi_{1}^{2} \xi_{2}^{4}+160 \tau_{1}^{2}|\tau|^{2} \xi_{1}^{2} \xi_{2}^{4}+16|\tau|^{4} \xi_{1}^{2} \xi_{2}^{4} \\
& \left.+64 \tau_{1}^{3}|\tau|^{2} \xi_{1} \xi_{2}^{5}+32 \tau_{1}|\tau|^{4} \xi_{1} \xi_{2}^{5}+16 \tau_{1}^{2}|\tau|^{4} \xi_{2}^{6}\right) b_{0}^{4} k^{5} \delta_{1}(k) b_{0} k \delta_{1}(k) \\
& +\left(8 \xi_{1}^{6}+48 \tau_{1} \xi_{1}^{5} \xi_{2}+104 \tau_{1}^{2} \xi_{1}^{4} \xi_{2}^{2}+16|\tau|^{2} \xi_{1}^{4} \xi_{2}^{2}+96 \tau_{1}^{3} \xi_{1}^{3} \xi_{2}^{3}\right. \\
& +64 \tau_{1}|\tau|^{2} \xi_{1}^{3} \xi_{2}^{3}+32 \tau_{1}^{4} \xi_{1}^{2} \xi_{2}^{4}+80 \tau_{1}^{2}|\tau|^{2} \xi_{1}^{2} \xi_{2}^{4}+8|\tau|^{4} \xi_{1}^{2} \xi_{2}^{4} \\
& \left.+32 \tau_{1}^{3}|\tau|^{2} \xi_{1} \xi_{2}^{5}+16 \tau_{1}|\tau|^{4} \xi_{1} \xi_{2}^{5}+8 \tau_{1}^{2}|\tau|^{4} \xi_{2}^{6}\right) b_{0}^{4} k^{4} \delta_{1}(k) b_{0} k^{2} \delta_{1}(k) \\
& +\left(4 \xi_{1}^{6}+(24) \tau_{1} \xi_{1}^{5} \xi_{2}+52 \tau_{1}^{2} \xi_{1}^{4} \xi_{2}^{2}+8|\tau|^{2} \xi_{1}^{4} \xi_{2}^{2}+48 \tau_{1}^{3} \xi_{1}^{3} \xi_{2}^{3}\right. \\
& +32 \tau_{1}|\tau|^{2} \xi_{1}^{3} \xi_{2}^{3}+16 \tau_{1}^{4} \xi_{1}^{2} \xi_{2}^{4}+40 \tau_{1}^{2}|\tau|^{2} \xi_{1}^{2} \xi_{2}^{4}+4|\tau|^{4} \xi_{1}^{2} \xi_{2}^{4} \\
& \left.+16 \tau_{1}^{3}|\tau|^{2} \xi_{1} \xi_{2}^{5}+8 \tau_{1}|\tau|^{4} \xi_{1} \xi_{2}^{5}+4 \tau_{1}^{2}|\tau|^{4} \xi_{2}^{6}\right) b_{0}^{3} k^{4} \delta_{1}(k) b_{0}^{2} k^{2} \delta_{1}(k) \\
& +\left(8 \xi_{1}^{6}+48 \tau_{1} \xi_{1}^{5} \xi_{2}+104 \tau_{1}^{2} \xi_{1}^{4} \xi_{2}^{2}+16|\tau|^{2} \xi_{1}^{4} \xi_{2}^{2}+96 \tau_{1}^{3} \xi_{1}^{3} \xi_{2}^{3}\right. \\
& +64 \tau_{1}|\tau|^{2} \xi_{1}^{3} \xi_{2}^{3}+32 \tau_{1}^{4} \xi_{1}^{2} \xi_{2}^{4}+80 \tau_{1}^{2}|\tau|^{2} \xi_{1}^{2} \xi_{2}^{4}+8|\tau|^{4} \xi_{1}^{2} \xi_{2}^{4} \\
& \left.+32 \tau_{1}^{3}|\tau|^{2} \xi_{1} \xi_{2}^{5}+16 \tau_{1}|\tau|^{4} \xi_{1} \xi_{2}^{5}+8 \tau_{1}^{2}|\tau|^{4} \xi_{2}^{6}\right) b_{0}^{3} k^{3} \delta_{1}(k) b_{0}^{2} k^{3} \delta_{1}(k) \\
& +\left(4 \xi_{1}^{6}+(24) \tau_{1} \xi_{1}^{5} \xi_{2}+52 \tau_{1}^{2} \xi_{1}^{4} \xi_{2}^{2}+8|\tau|^{2} \xi_{1}^{4} \xi_{2}^{2}+48 \tau_{1}^{3} \xi_{1}^{3} \xi_{2}^{3}\right. \\
& +32 \tau_{1}|\tau|^{2} \xi_{1}^{3} \xi_{2}^{3}+16 \tau_{1}^{4} \xi_{1}^{2} \xi_{2}^{4}+40 \tau_{1}^{2}|\tau|^{2} \xi_{1}^{2} \xi_{2}^{4}+4|\tau|^{4} \xi_{1}^{2} \xi_{2}^{4} \\
& \left.+16 \tau_{1}^{3}|\tau|^{2} \xi_{1} \xi_{2}^{5}+8 \tau_{1}|\tau|^{4} \xi_{1} \xi_{2}^{5}+4 \tau_{1}^{2}|\tau|^{4} \xi_{2}^{6}\right) b_{0}^{3} k^{2} \delta_{1}(k) b_{0}^{2} k^{4} \delta_{1}(k) \\
& +\left(8 \tau_{1} \xi_{1}^{6}+40 \tau_{1}^{2} \xi_{1}^{5} \xi_{2}+8|\tau|^{2} \xi_{1}^{5} \xi_{2}+64 \tau_{1}^{3} \xi_{1}^{4} \xi_{2}^{2}+56 \tau_{1}|\tau|^{2} \xi_{1}^{4} \xi_{2}^{2}\right. \\
& +32 \tau_{1}^{4} \xi_{1}^{3} \xi_{2}^{3}+112 \tau_{1}^{2}|\tau|^{2} \xi_{1}^{3} \xi_{2}^{3}+16|\tau|^{4} \xi_{1}^{3} \xi_{2}^{3}+64 \tau_{1}^{3}|\tau|^{2} \xi_{1}^{2} \xi_{2}^{4} \\
& \left.+56 \tau_{1}|\tau|^{4} \xi_{1}^{2} \xi_{2}^{4}+40 \tau_{1}^{2}|\tau|^{4} \xi_{1} \xi_{2}^{5}+8|\tau|^{6} \xi_{1} \xi_{2}^{5}+8 \tau_{1}|\tau|^{6} \xi_{2}^{6}\right) b_{0}^{4} k^{6} \delta_{1}(k) b_{0} \delta_{2}(k) \\
& +\left(8 \tau_{1} \xi_{1}^{6}+40 \tau_{1}^{2} \xi_{1}^{5} \xi_{2}+8|\tau|^{2} \xi_{1}^{5} \xi_{2}+64 \tau_{1}^{3} \xi_{1}^{4} \xi_{2}^{2}+56 \tau_{1}|\tau|^{2} \xi_{1}^{4} \xi_{2}^{2}\right. \\
& +32 \tau_{1}^{4} \xi_{1}^{3} \xi_{2}^{3}+112 \tau_{1}^{2}|\tau|^{2} \xi_{1}^{3} \xi_{2}^{3}+16|\tau|^{4} \xi_{1}^{3} \xi_{2}^{3}+64 \tau_{1}^{3}|\tau|^{2} \xi_{1}^{2} \xi_{2}^{4} \\
& \left.+56 \tau_{1}|\tau|^{4} \xi_{1}^{2} \xi_{2}^{4}+40 \tau_{1}^{2}|\tau|^{4} \xi_{1} \xi_{2}^{5}+8|\tau|^{6} \xi_{1} \xi_{2}^{5}+8 \tau_{1}|\tau|^{6} \xi_{2}^{6}\right) b_{0}^{4} k^{6} \delta_{2}(k) b_{0} \delta_{1}(k) \\
& +\left(16 \tau_{1} \xi_{1}^{6}+80 \tau_{1}^{2} \xi_{1}^{5} \xi_{2}+16|\tau|^{2} \xi_{1}^{5} \xi_{2}+128 \tau_{1}^{3} \xi_{1}^{4} \xi_{2}^{2}+112 \tau_{1}|\tau|^{2} \xi_{1}^{4} \xi_{2}^{2}\right. \\
& +64 \tau_{1}^{4} \xi_{1}^{3} \xi_{2}^{3}+224 \tau_{1}^{2}|\tau|^{2} \xi_{1}^{3} \xi_{2}^{3}+32|\tau|^{4} \xi_{1}^{3} \xi_{2}^{3}+128 \tau_{1}^{3}|\tau|^{2} \xi_{1}^{2} \xi_{2}^{4}+112 \tau_{1}|\tau|^{4} \xi_{1}^{2} \xi_{2}^{4} \\
& \left.+80 \tau_{1}^{2}|\tau|^{4} \xi_{1} \xi_{2}^{5}+16|\tau|^{6} \xi_{1} \xi_{2}^{5}+16 \tau_{1}|\tau|^{6} \xi_{2}^{6}\right) b_{0}^{4} k^{5} \delta_{1}(k) b_{0} k \delta_{2}(k)
\end{aligned}
$$




$$
\begin{aligned}
& +\left(16 \tau_{1} \xi_{1}^{6}+80 \tau_{1}^{2} \xi_{1}^{5} \xi_{2}+16|\tau|^{2} \xi_{1}^{5} \xi_{2}+128 \tau_{1}^{3} \xi_{1}^{4} \xi_{2}^{2}+112 \tau_{1}|\tau|^{2} \xi_{1}^{4} \xi_{2}^{2}\right. \\
& +64 \tau_{1}^{4} \xi_{1}^{3} \xi_{2}^{3}+224 \tau_{1}^{2}|\tau|^{2} \xi_{1}^{3} \xi_{2}^{3}+32|\tau|^{4} \xi_{1}^{3} \xi_{2}^{3}+128 \tau_{1}^{3}|\tau|^{2} \xi_{1}^{2} \xi_{2}^{4} \\
& \left.+112 \tau_{1}|\tau|^{4} \xi_{1}^{2} \xi_{2}^{4}+80 \tau_{1}^{2}|\tau|^{4} \xi_{1} \xi_{2}^{5}+16|\tau|^{6} \xi_{1} \xi_{2}^{5}+16 \tau_{1}|\tau|^{6} \xi_{2}^{6}\right) b_{0}^{4} k^{5} \delta_{2}(k) b_{0} k \delta_{1}(k) \\
& +\left(8 \tau_{1} \xi_{1}^{6}+40 \tau_{1}^{2} \xi_{1}^{5} \xi_{2}+8|\tau|^{2} \xi_{1}^{5} \xi_{2}+64 \tau_{1}^{3} \xi_{1}^{4} \xi_{2}^{2}+56 \tau_{1}|\tau|^{2} \xi_{1}^{4} \xi_{2}^{2}\right. \\
& +32 \tau_{1}^{4} \xi_{1}^{3} \xi_{2}^{3}+112 \tau_{1}^{2}|\tau|^{2} \xi_{1}^{3} \xi_{2}^{3}+16|\tau|^{4} \xi_{1}^{3} \xi_{2}^{3}+64 \tau_{1}^{3}|\tau|^{2} \xi_{1}^{2} \xi_{2}^{4} \\
& \left.+56 \tau_{1}|\tau|^{4} \xi_{1}^{2} \xi_{2}^{4}+40 \tau_{1}^{2}|\tau|^{4} \xi_{1} \xi_{2}^{5}+8|\tau|^{6} \xi_{1} \xi_{2}^{5}+8 \tau_{1}|\tau|^{6} \xi_{2}^{6}\right) b_{0}^{4} k^{4} \delta_{1}(k) b_{0} k^{2} \delta_{2}(k) \\
& +\left(8 \tau_{1} \xi_{1}^{6}+40 \tau_{1}^{2} \xi_{1}^{5} \xi_{2}+8|\tau|^{2} \xi_{1}^{5} \xi_{2}+64 \tau_{1}^{3} \xi_{1}^{4} \xi_{2}^{2}+56 \tau_{1}|\tau|^{2} \xi_{1}^{4} \xi_{2}^{2}\right. \\
& +32 \tau_{1}^{4} \xi_{1}^{3} \xi_{2}^{3}+112 \tau_{1}^{2}|\tau|^{2} \xi_{1}^{3} \xi_{2}^{3}+16|\tau|^{4} \xi_{1}^{3} \xi_{2}^{3}+64 \tau_{1}^{3}|\tau|^{2} \xi_{1}^{2} \xi_{2}^{4} \\
& \left.+56 \tau_{1}|\tau|^{4} \xi_{1}^{2} \xi_{2}^{4}+40 \tau_{1}^{2}|\tau|^{4} \xi_{1} \xi_{2}^{5}+8|\tau|^{6} \xi_{1} \xi_{2}^{5}+8 \tau_{1}|\tau|^{6} \xi_{2}^{6}\right) b_{0}^{4} k^{4} \delta_{2}(k) b_{0} k^{2} \delta_{1}(k) \\
& +\left(4 \tau_{1} \xi_{1}^{6}+20 \tau_{1}^{2} \xi_{1}^{5} \xi_{2}+4|\tau|^{2} \xi_{1}^{5} \xi_{2}+32 \tau_{1}^{3} \xi_{1}^{4} \xi_{2}^{2}+28 \tau_{1}|\tau|^{2} \xi_{1}^{4} \xi_{2}^{2}\right. \\
& +16 \tau_{1}^{4} \xi_{1}^{3} \xi_{2}^{3}+56 \tau_{1}^{2}|\tau|^{2} \xi_{1}^{3} \xi_{2}^{3}+8|\tau|^{4} \xi_{1}^{3} \xi_{2}^{3}+32 \tau_{1}^{3}|\tau|^{2} \xi_{1}^{2} \xi_{2}^{4} \\
& \left.+28 \tau_{1}|\tau|^{4} \xi_{1}^{2} \xi_{2}^{4}+20 \tau_{1}^{2}|\tau|^{4} \xi_{1} \xi_{2}^{5}+4|\tau|^{6} \xi_{1} \xi_{2}^{5}+4 \tau_{1}|\tau|^{6} \xi_{2}^{6}\right) b_{0}^{3} k^{4} \delta_{1}(k) b_{0}^{2} k^{2} \delta_{2}(k) \\
& +\left(4 \tau_{1} \xi_{1}^{6}+20 \tau_{1}^{2} \xi_{1}^{5} \xi_{2}+4|\tau|^{2} \xi_{1}^{5} \xi_{2}+32 \tau_{1}^{3} \xi_{1}^{4} \xi_{2}^{2}+28 \tau_{1}|\tau|^{2} \xi_{1}^{4} \xi_{2}^{2}\right. \\
& +16 \tau_{1}^{4} \xi_{1}^{3} \xi_{2}^{3}+56 \tau_{1}^{2}|\tau|^{2} \xi_{1}^{3} \xi_{2}^{3}+8|\tau|^{4} \xi_{1}^{3} \xi_{2}^{3}+32 \tau_{1}^{3}|\tau|^{2} \xi_{1}^{2} \xi_{2}^{4} \\
& \left.+28 \tau_{1}|\tau|^{4} \xi_{1}^{2} \xi_{2}^{4}+20 \tau_{1}^{2}|\tau|^{4} \xi_{1} \xi_{2}^{5}+4|\tau|^{6} \xi_{1} \xi_{2}^{5}+4 \tau_{1}|\tau|^{6} \xi_{2}^{6}\right) b_{0}^{3} k^{4} \delta_{2}(k) b_{0}^{2} k^{2} \delta_{1}(k) \\
& +\left(8 \tau_{1} \xi_{1}^{6}+40 \tau_{1}^{2} \xi_{1}^{5} \xi_{2}+8|\tau|^{2} \xi_{1}^{5} \xi_{2}+64 \tau_{1}^{3} \xi_{1}^{4} \xi_{2}^{2}+56 \tau_{1}|\tau|^{2} \xi_{1}^{4} \xi_{2}^{2}\right. \\
& +32 \tau_{1}^{4} \xi_{1}^{3} \xi_{2}^{3}+112 \tau_{1}^{2}|\tau|^{2} \xi_{1}^{3} \xi_{2}^{3}+16|\tau|^{4} \xi_{1}^{3} \xi_{2}^{3}+64 \tau_{1}^{3}|\tau|^{2} \xi_{1}^{2} \xi_{2}^{4} \\
& \left.+56 \tau_{1}|\tau|^{4} \xi_{1}^{2} \xi_{2}^{4}+40 \tau_{1}^{2}|\tau|^{4} \xi_{1} \xi_{2}^{5}+8|\tau|^{6} \xi_{1} \xi_{2}^{5}+8 \tau_{1}|\tau|^{6} \xi_{2}^{6}\right) b_{0}^{3} k^{3} \delta_{1}(k) b_{0}^{2} k^{3} \delta_{2}(k) \\
& +\left(8 \tau_{1} \xi_{1}^{6}+40 \tau_{1}^{2} \xi_{1}^{5} \xi_{2}+8|\tau|^{2} \xi_{1}^{5} \xi_{2}+64 \tau_{1}^{3} \xi_{1}^{4} \xi_{2}^{2}+56 \tau_{1}|\tau|^{2} \xi_{1}^{4} \xi_{2}^{2}\right. \\
& +32 \tau_{1}^{4} \xi_{1}^{3} \xi_{2}^{3}+112 \tau_{1}^{2}|\tau|^{2} \xi_{1}^{3} \xi_{2}^{3}+16|\tau|^{4} \xi_{1}^{3} \xi_{2}^{3}+64 \tau_{1}^{3}|\tau|^{2} \xi_{1}^{2} \xi_{2}^{4} \\
& \left.+56 \tau_{1}|\tau|^{4} \xi_{1}^{2} \xi_{2}^{4}+40 \tau_{1}^{2}|\tau|^{4} \xi_{1} \xi_{2}^{5}+8|\tau|^{6} \xi_{1} \xi_{2}^{5}+8 \tau_{1}|\tau|^{6} \xi_{2}^{6}\right) b_{0}^{3} k^{3} \delta_{2}(k) b_{0}^{2} k^{3} \delta_{1}(k) \\
& +\left(4 \tau_{1} \xi_{1}^{6}+20 \tau_{1}^{2} \xi_{1}^{5} \xi_{2}+4|\tau|^{2} \xi_{1}^{5} \xi_{2}+32 \tau_{1}^{3} \xi_{1}^{4} \xi_{2}^{2}+28 \tau_{1}|\tau|^{2} \xi_{1}^{4} \xi_{2}^{2}\right. \\
& +16 \tau_{1}^{4} \xi_{1}^{3} \xi_{2}^{3}+56 \tau_{1}^{2}|\tau|^{2} \xi_{1}^{3} \xi_{2}^{3}+8|\tau|^{4} \xi_{1}^{3} \xi_{2}^{3}+32 \tau_{1}^{3}|\tau|^{2} \xi_{1}^{2} \xi_{2}^{4} \\
& \left.+28 \tau_{1}|\tau|^{4} \xi_{1}^{2} \xi_{2}^{4}+20 \tau_{1}^{2}|\tau|^{4} \xi_{1} \xi_{2}^{5}+4|\tau|^{6} \xi_{1} \xi_{2}^{5}+4 \tau_{1}|\tau|^{6} \xi_{2}^{6}\right) b_{0}^{3} k^{2} \delta_{1}(k) b_{0}^{2} k^{4} \delta_{2}(k) \\
& +\left(4 \tau_{1} \xi_{1}^{6}+20 \tau_{1}^{2} \xi_{1}^{5} \xi_{2}+4|\tau|^{2} \xi_{1}^{5} \xi_{2}+32 \tau_{1}^{3} \xi_{1}^{4} \xi_{2}^{2}+28 \tau_{1}|\tau|^{2} \xi_{1}^{4} \xi_{2}^{2}\right. \\
& +16 \tau_{1}^{4} \xi_{1}^{3} \xi_{2}^{3}+56 \tau_{1}^{2}|\tau|^{2} \xi_{1}^{3} \xi_{2}^{3}+8|\tau|^{4} \xi_{1}^{3} \xi_{2}^{3}+32 \tau_{1}^{3}|\tau|^{2} \xi_{1}^{2} \xi_{2}^{4} \\
& \left.+28 \tau_{1}|\tau|^{4} \xi_{1}^{2} \xi_{2}^{4}+20 \tau_{1}^{2}|\tau|^{4} \xi_{1} \xi_{2}^{5}+4|\tau|^{6} \xi_{1} \xi_{2}^{5}+4 \tau_{1}|\tau|^{6} \xi_{2}^{6}\right) b_{0}^{3} k^{2} \delta_{2}(k) b_{0}^{2} k^{4} \delta_{1}(k) \\
& +\left(8 \tau_{1}^{2} \xi_{1}^{6}+32 \tau_{1}^{3} \xi_{1}^{5} \xi_{2}+16 \tau_{1}|\tau|^{2} \xi_{1}^{5} \xi_{2}+32 \tau_{1}^{4} \xi_{1}^{4} \xi_{2}^{2}\right. \\
& +80 \tau_{1}^{2}|\tau|^{2} \xi_{1}^{4} \xi_{2}^{2}+8|\tau|^{4} \xi_{1}^{4} \xi_{2}^{2}+96 \tau_{1}^{3}|\tau|^{2} \xi_{1}^{3} \xi_{2}^{3}+64 \tau_{1}|\tau|^{4} \xi_{1}^{3} \xi_{2}^{3} \\
& \left.+104 \tau_{1}^{2}|\tau|^{4} \xi_{1}^{2} \xi_{2}^{4}+16|\tau|^{6} \xi_{1}^{2} \xi_{2}^{4}+48 \tau_{1}|\tau|^{6} \xi_{1} \xi_{2}^{5}+8|\tau|^{8} \xi_{2}^{6}\right) b_{0}^{4} k^{6} \delta_{2}(k) b_{0} \delta_{2}(k) \\
& +\left(16 \tau_{1}^{2} \xi_{1}^{6}+64 \tau_{1}^{3} \xi_{1}^{5} \xi_{2}+32 \tau_{1}|\tau|^{2} \xi_{1}^{5} \xi_{2}+64 \tau_{1}^{4} \xi_{1}^{4} \xi_{2}^{2}\right. \\
& +160 \tau_{1}^{2}|\tau|^{2} \xi_{1}^{4} \xi_{2}^{2}+16|\tau|^{4} \xi_{1}^{4} \xi_{2}^{2}+192 \tau_{1}^{3}|\tau|^{2} \xi_{1}^{3} \xi_{2}^{3}+128 \tau_{1}|\tau|^{4} \xi_{1}^{3} \xi_{2}^{3} \\
& \left.+208 \tau_{1}^{2}|\tau|^{4} \xi_{1}^{2} \xi_{2}^{4}+32|\tau|^{6} \xi_{1}^{2} \xi_{2}^{4}+96 \tau_{1}|\tau|^{6} \xi_{1} \xi_{2}^{5}+16|\tau|^{8} \xi_{2}^{6}\right) b_{0}^{4} k^{5} \delta_{2}(k) b_{0} k \delta_{2}(k)
\end{aligned}
$$


Gauss-Bonnet theorem for noncommutative 2-tori with a general conformal structure 469

$$
\begin{aligned}
& +\left(8 \tau_{1}^{2} \xi_{1}^{6}+32 \tau_{1}^{3} \xi_{1}^{5} \xi_{2}+16 \tau_{1}|\tau|^{2} \xi_{1}^{5} \xi_{2}+32 \tau_{1}^{4} \xi_{1}^{4} \xi_{2}^{2}\right. \\
& +80 \tau_{1}^{2}|\tau|^{2} \xi_{1}^{4} \xi_{2}^{2}+8|\tau|^{4} \xi_{1}^{4} \xi_{2}^{2}+96 \tau_{1}^{3}|\tau|^{2} \xi_{1}^{3} \xi_{2}^{3}+64 \tau_{1}|\tau|^{4} \xi_{1}^{3} \xi_{2}^{3} \\
& \left.+104 \tau_{1}^{2}|\tau|^{4} \xi_{1}^{2} \xi_{2}^{4}+16|\tau|^{6} \xi_{1}^{2} \xi_{2}^{4}+48 \tau_{1}|\tau|^{6} \xi_{1} \xi_{2}^{5}+8|\tau|^{8} \xi_{2}^{6}\right) b_{0}^{4} k^{4} \delta_{2}(k) b_{0} k^{2} \delta_{2}(k) \\
& +\left(4 \tau_{1}^{2} \xi_{1}^{6}+16 \tau_{1}^{3} \xi_{1}^{5} \xi_{2}+8 \tau_{1}|\tau|^{2} \xi_{1}^{5} \xi_{2}+16 \tau_{1}^{4} \xi_{1}^{4} \xi_{2}^{2}\right. \\
& +40 \tau_{1}^{2}|\tau|^{2} \xi_{1}^{4} \xi_{2}^{2}+4|\tau|^{4} \xi_{1}^{4} \xi_{2}^{2}+48 \tau_{1}^{3}|\tau|^{2} \xi_{1}^{3} \xi_{2}^{3}+32 \tau_{1}|\tau|^{4} \xi_{1}^{3} \xi_{2}^{3} \\
& \left.+52 \tau_{1}^{2}|\tau|^{4} \xi_{1}^{2} \xi_{2}^{4}+8|\tau|^{6} \xi_{1}^{2} \xi_{2}^{4}+(24) \tau_{1}|\tau|^{6} \xi_{1} \xi_{2}^{5}+4|\tau|^{8} \xi_{2}^{6}\right) b_{0}^{3} k^{4} \delta_{2}(k) b_{0}^{2} k^{2} \delta_{2}(k) \\
& +\left(8 \tau_{1}^{2} \xi_{1}^{6}+32 \tau_{1}^{3} \xi_{1}^{5} \xi_{2}+16 \tau_{1}|\tau|^{2} \xi_{1}^{5} \xi_{2}+32 \tau_{1}^{4} \xi_{1}^{4} \xi_{2}^{2}\right. \\
& +80 \tau_{1}^{2}|\tau|^{2} \xi_{1}^{4} \xi_{2}^{2}+8|\tau|^{4} \xi_{1}^{4} \xi_{2}^{2}+96 \tau_{1}^{3}|\tau|^{2} \xi_{1}^{3} \xi_{2}^{3}+64 \tau_{1}|\tau|^{4} \xi_{1}^{3} \xi_{2}^{3} \\
& \left.+104 \tau_{1}^{2}|\tau|^{4} \xi_{1}^{2} \xi_{2}^{4}+16|\tau|^{6} \xi_{1}^{2} \xi_{2}^{4}+48 \tau_{1}|\tau|^{6} \xi_{1} \xi_{2}^{5}+8|\tau|^{8} \xi_{2}^{6}\right) b_{0}^{3} k^{3} \delta_{2}(k) b_{0}^{2} k^{3} \delta_{2}(k) \\
& +\left(4 \tau_{1}^{2} \xi_{1}^{6}+16 \tau_{1}^{3} \xi_{1}^{5} \xi_{2}+8 \tau_{1}|\tau|^{2} \xi_{1}^{5} \xi_{2}+16 \tau_{1}^{4} \xi_{1}^{4} \xi_{2}^{2}\right. \\
& +40 \tau_{1}^{2}|\tau|^{2} \xi_{1}^{4} \xi_{2}^{2}+4|\tau|^{4} \xi_{1}^{4} \xi_{2}^{2}+48 \tau_{1}^{3}|\tau|^{2} \xi_{1}^{3} \xi_{2}^{3}+32 \tau_{1}|\tau|^{4} \xi_{1}^{3} \xi_{2}^{3} \\
& \left.+52 \tau_{1}^{2}|\tau|^{4} \xi_{1}^{2} \xi_{2}^{4}+8|\tau|^{6} \xi_{1}^{2} \xi_{2}^{4}+(24) \tau \tau_{1}|\tau|^{6} \xi_{1} \xi_{2}^{5}+4|\tau|^{8} \xi_{2}^{6}\right) b_{0}^{3} k^{2} \delta_{2}(k) b_{0}^{2} k^{4} \delta_{2}(k) .
\end{aligned}
$$

To integrate these terms over the $\xi$-plane we pass to the coordinates

$$
\xi_{1}=r \cos \theta-r \frac{\tau_{1}}{\tau_{2}} \sin \theta, \quad \xi_{2}=\frac{r}{\tau_{2}} \sin \theta,
$$

where $\theta$ ranges from 0 to $2 \pi$ and $r$ ranges from 0 to $\infty$.

After multiplication by the Jacobian of the change of coordinates, which is equal to $\frac{r}{\tau_{2}}$, and integrating with respect to $\theta$ from 0 to $2 \pi$, one gets, up to an overall factor of $\frac{2 \pi}{\tau_{2}}$ :

$$
\begin{aligned}
& -r b_{0}^{2} k \delta_{1}^{2}(k)-2 \tau_{1} r b_{0}^{2} k \delta_{1} \delta_{2}(k)-|\tau|^{2} r b_{0}^{2} k \delta_{2}^{2}(k)+4 r^{3} b_{0}^{3} k^{3} \delta_{1}^{2}(k) \\
& +4 r^{3} b_{0}^{3} k^{2} \delta_{1}(k)^{2}+2 r^{3} b_{0}^{2} k^{2} \delta_{1}(k) b_{0} \delta_{1}(k)+4 r^{3} b_{0}^{2} k \delta_{1}(k) b_{0} k \delta_{1}(k) \\
& +8 \tau_{1} r^{3} b_{0}^{3} k^{3} \delta_{1} \delta_{2}(k)+4 \tau_{1} r^{3} b_{0}^{3} k^{2} \delta_{1}(k) \delta_{2}(k)+4 \tau_{1} r^{3} b_{0}^{3} k^{2} \delta_{2}(k) \delta_{1}(k) \\
& +2 \tau_{1} r^{3} b_{0}^{2} k^{2} \delta_{1}(k) b_{0} \delta_{2}(k)+2 \tau_{1} r^{3} b_{0}^{2} k^{2} \delta_{2}(k) b_{0} \delta_{1}(k)+4 \tau_{1} r^{3} b_{0}^{2} k \delta_{1}(k) b_{0} k \delta_{2}(k) \\
& +4 \tau_{1} r^{3} b_{0}^{2} k \delta_{2}(k) b_{0} k \delta_{1}(k)+4|\tau|^{2} r^{3} b_{0}^{3} k^{3} \delta_{2}^{2}(k)+4|\tau|^{2} r^{3} b_{0}^{3} k^{2} \delta_{2}(k)^{2} \\
& +4|\tau|^{2} r^{3} b_{0}^{2} k \delta_{2}(k) b_{0} k \delta_{2}(k)+2|\tau|^{2} r^{3} b_{0}^{2} k^{2} \delta_{2}(k) b_{0} \delta_{2}(k)-4 r^{5} b_{0}^{4} k^{5} \delta_{1}^{2}(k) \\
& -4 r^{5} b_{0}^{4} k^{4} \delta_{1}(k)^{2}-6 r^{5} b_{0}^{3} k^{4} \delta_{1}(k) b_{0} \delta_{1}(k)-12 r^{5} b_{0}^{3} k^{3} \delta_{1}(k) b_{0} k \delta_{1}(k) \\
& -6 r^{5} b_{0}^{3} k^{2} \delta_{1}(k) b_{0} k^{2} \delta_{1}(k)-2 r^{5} b_{0}^{2} k^{2} \delta_{1}(k) b_{0}^{2} k^{2} \delta_{1}(k)-2 r^{5} b_{0}^{2} k \delta_{1}(k) b_{0}^{2} k^{3} \delta_{1}(k) \\
& -8 \tau_{1} r^{5} b_{0}^{4} k^{5} \delta_{1} \delta_{2}(k)-4 \tau_{1} r^{5} b_{0}^{4} k^{4} \delta_{1}(k) \delta_{2}(k)-4 \tau_{1} r^{5} b_{0}^{4} k^{4} \delta_{2}(k) \delta_{1}(k) \\
& -6 \tau_{1} r^{5} b_{0}^{3} k^{4} \delta_{1}(k) b_{0} \delta_{2}(k)-6 \tau_{1} r^{5} b_{0}^{3} k^{4} \delta_{2}(k) b_{0} \delta_{1}(k) \\
& -12 \tau_{1} r^{5} b_{0}^{3} k^{3} \delta_{1}(k) b_{0} k \delta_{2}(k)-12 \tau_{1} r^{5} b_{0}^{3} k^{3} \delta_{2}(k) b_{0} k \delta_{1}(k) \\
& -6 \tau_{1} r^{5} b_{0}^{3} k^{2} \delta_{1}(k) b_{0} k^{2} \delta_{2}(k)-6 \tau_{1} r^{5} b_{0}^{3} k^{2} \delta_{2}(k) b_{0} k^{2} \delta_{1}(k) \\
& -4 \tau_{1} r^{5} b_{0}^{2} k^{2} \delta_{1}(k) b_{0}^{2} k^{2} \delta_{2}(k)-2 \tau_{1} r^{5} b_{0}^{2} k \delta_{1}(k) b_{0}^{2} k^{3} \delta_{2}(k)
\end{aligned}
$$




$$
\begin{aligned}
& -2 \tau_{1} r^{5} b_{0}^{2} k \delta_{2}(k) b_{0}^{2} k^{3} \delta_{1}(k)-4|\tau|^{2} r^{5} b_{0}^{4} k^{5} \delta_{2}^{2}(k)-4|\tau|^{2} r^{5} b_{0}^{4} k^{4} \delta_{2}(k)^{2} \\
& -6|\tau|^{2} r^{5} b_{0}^{3} k^{4} \delta_{2}(k) b_{0} \delta_{2}(k)-12|\tau|^{2} r^{5} b_{0}^{3} k^{3} \delta_{2}(k) b_{0} k \delta_{2}(k) \\
& -6|\tau|^{2} r^{5} b_{0}^{3} k^{2} \delta_{2}(k) b_{0} k^{2} \delta_{2}(k)-2|\tau|^{2} r^{5} b_{0}^{2} k^{2} \delta_{2}(k) b_{0}^{2} k^{2} \delta_{2}(k) \\
& -2|\tau|^{2} r^{5} b_{0}^{2} k \delta_{2}(k) b_{0}^{2} k^{3} \delta_{2}(k)+4 r^{7} b_{0}^{4} k^{6} \delta_{1}(k) b_{0} \delta_{1}(k)+8 r^{7} b_{0}^{4} k^{5} \delta_{1}(k) b_{0} k \delta_{1}(k) \\
& +4 r^{7} b_{0}^{4} k^{4} \delta_{1}(k) b_{0} k^{2} \delta_{1}(k)+2 r^{7} b_{0}^{3} k^{4} \delta_{1}(k) b_{0}^{2} k^{2} \delta_{1}(k) \\
& +4 r^{7} b_{0}^{3} k^{3} \delta_{1}(k) b_{0}^{2} k^{3} \delta_{1}(k)+2 r^{7} b_{0}^{3} k^{2} \delta_{1}(k) b_{0}^{2} k^{4} \delta_{1}(k) \\
& +4 \tau_{1} r^{7} b_{0}^{4} k^{6} \delta_{1}(k) b_{0} \delta_{2}(k)+4 \tau_{1} r^{7} b_{0}^{4} k^{6} \delta_{2}(k) b_{0} \delta_{1}(k) \\
& +8 \tau_{1} r^{7} b_{0}^{4} k^{5} \delta_{1}(k) b_{0} k \delta_{2}(k)+8 \tau_{1} r^{7} b_{0}^{4} k^{5} \delta_{2}(k) b_{0} k \delta_{1}(k) \\
& +4 \tau_{1} r^{7} b_{0}^{4} k^{4} \delta_{1}(k) b_{0} k^{2} \delta_{2}(k)+4 \tau_{1} r^{7} b_{0}^{4} k^{4} \delta_{2}(k) b_{0} k^{2} \delta_{1}(k) \\
& +2 \tau_{1} r^{7} b_{0}^{3} k^{4} \delta_{1}(k) b_{0}^{2} k^{2} \delta_{2}(k)+2 \tau_{1} r^{7} b_{0}^{3} k^{4} \delta_{2}(k) b_{0}^{2} k^{2} \delta_{1}(k) \\
& +4 \tau_{1} r^{7} b_{0}^{3} k^{3} \delta_{1}(k) b_{0}^{2} k^{3} \delta_{2}(k)+4 \tau_{1} r^{7} b_{0}^{3} k^{3} \delta_{2}(k) b_{0}^{2} k^{3} \delta_{1}(k) \\
& +2 \tau_{1} r^{7} b_{0}^{3} k^{2} \delta_{1}(k) b_{0}^{2} k^{4} \delta_{2}(k)+2 \tau_{1} r^{7} b_{0}^{3} k^{2} \delta_{2}(k) b_{0}^{2} k^{4} \delta_{1}(k) \\
& +4|\tau|^{2} r^{7} b_{0}^{4} k^{6} \delta_{2}(k) b_{0} \delta_{2}(k)+8|\tau|^{2} r^{7} b_{0}^{4} k^{5} \delta_{2}(k) b_{0} k \delta_{2}(k) \\
& +4|\tau|^{2} r^{7} b_{0}^{4} k^{4} \delta_{2}(k) b_{0} k^{2} \delta_{2}(k)+2|\tau|^{2} r^{7} b_{0}^{3} k^{4} \delta_{2}(k) b_{0}^{2} k^{2} \delta_{2}(k) \\
& +4|\tau|^{2} r^{7} b_{0}^{3} k^{3} \delta_{2}(k) b_{0}^{2} k^{3} \delta_{2}(k)+2|\tau|^{2} r^{7} b_{0}^{3} k^{2} \delta_{2}(k) b_{0}^{2} k^{4} \delta_{2}(k),
\end{aligned}
$$

where

$$
b_{0}=\left(r^{2} k^{2}+1\right)^{-1} .
$$

4.1. Terms with all $\boldsymbol{b}_{\mathbf{0}}$ on the left. The integral $\int_{0}^{\infty} \bullet d r$ of these terms, namely

$$
\begin{aligned}
& -r b_{0}^{2} k \delta_{1}^{2}(k)-2 \tau_{1} r b_{0}^{2} k \delta_{1} \delta_{2}(k)-|\tau|^{2} r b_{0}^{2} k \delta_{2}^{2}(k)+4 r^{3} b_{0}^{3} k^{3} \delta_{1}^{2}(k) \\
& +4 r^{3} b_{0}^{3} k^{2} \delta_{1}(k)^{2}+8 \tau_{1} r^{3} b_{0}^{3} k^{3} \delta_{1} \delta_{2}(k)+4 \tau_{1} r^{3} b_{0}^{3} k^{2} \delta_{1}(k) \delta_{2}(k) \\
& +4 \tau_{1} r^{3} b_{0}^{3} k^{2} \delta_{2}(k) \delta_{1}(k)+4|\tau|^{2} r^{3} b_{0}^{3} k^{3} \delta_{2}^{2}(k)+4|\tau|^{2} r^{3} b_{0}^{3} k^{2} \delta_{2}(k)^{2} \\
& -4 r^{5} b_{0}^{4} k^{5} \delta_{1}^{2}(k)-4 r^{5} b_{0}^{4} k^{4} \delta_{1}(k)^{2}-8 \tau_{1} r^{5} b_{0}^{4} k^{5} \delta_{1} \delta_{2}(k) \\
& -4 \tau_{1} r^{5} b_{0}^{4} k^{4} \delta_{1}(k) \delta_{2}(k)-4 \tau_{1} r^{5} b_{0}^{4} k^{4} \delta_{2}(k) \delta_{1}(k) \\
& -4|\tau|^{2} r^{5} b_{0}^{4} k^{5} \delta_{2}^{2}(k)-4|\tau|^{2} r^{5} b_{0}^{4} k^{4} \delta_{2}(k)^{2}
\end{aligned}
$$

gives (up to an overall factor of $\frac{2 \pi}{\tau_{2}}$ )

$$
\begin{array}{r}
-\frac{1}{6} k^{-1} \delta_{1}^{2}(k)-\frac{|\tau|^{2}}{6} k^{-1} \delta_{2}^{2}(k)-\frac{\tau_{1}}{3} k^{-1} \delta_{1} \delta_{2}(k)+\frac{1}{3} k^{-2} \delta_{1}(k)^{2} \\
+\frac{|\tau|^{2}}{3} k^{-2} \delta_{2}(k)^{2}+\frac{\tau_{1}}{3} k^{-2} \delta_{1}(k) \delta_{2}(k)+\frac{\tau_{1}}{3} k^{-2} \delta_{2}(k) \delta_{1}(k) .
\end{array}
$$


Gauss-Bonnet theorem for noncommutative 2-tori with a general conformal structure 471

4.2. Terms with $b_{0}$ in the middle. They are the following terms:

$$
\begin{aligned}
& 2 r^{3} b_{0}^{2} k^{2} \delta_{1}(k) b_{0} \delta_{1}(k)+4 r^{3} b_{0}^{2} k \delta_{1}(k) b_{0} k \delta_{1}(k)+2 \tau_{1} r^{3} b_{0}^{2} k^{2} \delta_{1}(k) b_{0} \delta_{2}(k) \\
& +2 \tau_{1} r^{3} b_{0}^{2} k^{2} \delta_{2}(k) b_{0} \delta_{1}(k)+4 \tau_{1} r^{3} b_{0}^{2} k \delta_{1}(k) b_{0} k \delta_{2}(k)+4 \tau_{1} r^{3} b_{0}^{2} k \delta_{2}(k) b_{0} k \delta_{1}(k) \\
& +4|\tau|^{2} r^{3} b_{0}^{2} k \delta_{2}(k) b_{0} k \delta_{2}(k)+2|\tau|^{2} r^{3} b_{0}^{2} k^{2} \delta_{2}(k) b_{0} \delta_{2}(k)-6 r^{5} b_{0}^{3} k^{4} \delta_{1}(k) b_{0} \delta_{1}(k) \\
& -12 r^{5} b_{0}^{3} k^{3} \delta_{1}(k) b_{0} k \delta_{1}(k)-6 r^{5} b_{0}^{3} k^{2} \delta_{1}(k) b_{0} k^{2} \delta_{1}(k)-6 \tau_{1} r^{5} b_{0}^{3} k^{4} \delta_{1}(k) b_{0} \delta_{2}(k) \\
& -6 \tau_{1} r^{5} b_{0}^{3} k^{4} \delta_{2}(k) b_{0} \delta_{1}(k)-12 \tau_{1} r^{5} b_{0}^{3} k^{3} \delta_{1}(k) b_{0} k \delta_{2}(k) \\
& -12 \tau_{1} r^{5} b_{0}^{3} k^{3} \delta_{2}(k) b_{0} k \delta_{1}(k)-6 \tau_{1} r^{5} b_{0}^{3} k^{2} \delta_{1}(k) b_{0} k^{2} \delta_{2}(k) \\
& -6 \tau_{1} r^{5} b_{0}^{3} k^{2} \delta_{2}(k) b_{0} k^{2} \delta_{1}(k)-6|\tau|^{2} r^{5} b_{0}^{3} k^{4} \delta_{2}(k) b_{0} \delta_{2}(k) \\
& -12|\tau|^{2} r^{5} b_{0}^{3} k^{3} \delta_{2}(k) b_{0} k \delta_{2}(k)-6|\tau|^{2} r^{5} b_{0}^{3} k^{2} \delta_{2}(k) b_{0} k^{2} \delta_{2}(k) \\
& +4 r^{7} b_{0}^{4} k^{6} \delta_{1}(k) b_{0} \delta_{1}(k)+8 r^{7} b_{0}^{4} k^{5} \delta_{1}(k) b_{0} k \delta_{1}(k) \\
& +4 r^{7} b_{0}^{4} k^{4} \delta_{1}(k) b_{0} k^{2} \delta_{1}(k)+4 \tau_{1} r^{7} b_{0}^{4} k^{6} \delta_{1}(k) b_{0} \delta_{2}(k) \\
& +4 \tau_{1} r^{7} b_{0}^{4} k^{6} \delta_{2}(k) b_{0} \delta_{1}(k)+8 \tau_{1} r^{7} b_{0}^{4} k^{5} \delta_{1}(k) b_{0} k \delta_{2}(k) \\
& +8 \tau_{1} r^{7} b_{0}^{4} k^{5} \delta_{2}(k) b_{0} k \delta_{1}(k)+4 \tau_{1} r^{7} b_{0}^{4} k^{4} \delta_{1}(k) b_{0} k^{2} \delta_{2}(k) \\
& +4 \tau_{1} r^{7} b_{0}^{4} k^{4} \delta_{2}(k) b_{0} k^{2} \delta_{1}(k)+4|\tau|^{2} r^{7} b_{0}^{4} k^{6} \delta_{2}(k) b_{0} \delta_{2}(k) \\
& +8|\tau|^{2} r^{7} b_{0}^{4} k^{5} \delta_{2}(k) b_{0} k \delta_{2}(k)+4|\tau|^{2} r^{7} b_{0}^{4} k^{4} \delta_{2}(k) b_{0} k^{2} \delta_{2}(k) .
\end{aligned}
$$

4.3. The rest of the terms (with $b_{0}^{2}$ in the middle). These terms, namely

$$
\begin{aligned}
& -2 r^{5} b_{0}^{2} k^{2} \delta_{1}(k) b_{0}^{2} k^{2} \delta_{1}(k)-2 r^{5} b_{0}^{2} k \delta_{1}(k) b_{0}^{2} k^{3} \delta_{1}(k) \\
& -4 \tau_{1} r^{5} b_{0}^{2} k^{2} \delta_{1}(k) b_{0}^{2} k^{2} \delta_{2}(k)-2 \tau_{1} r^{5} b_{0}^{2} k \delta_{1}(k) b_{0}^{2} k^{3} \delta_{2}(k) \\
& -2 \tau_{1} r^{5} b_{0}^{2} k \delta_{2}(k) b_{0}^{2} k^{3} \delta_{1}(k)-2|\tau|^{2} r^{5} b_{0}^{2} k^{2} \delta_{2}(k) b_{0}^{2} k^{2} \delta_{2}(k) \\
& -2|\tau|^{2} r^{5} b_{0}^{2} k \delta_{2}(k) b_{0}^{2} k^{3} \delta_{2}(k)+2 r^{7} b_{0}^{3} k^{4} \delta_{1}(k) b_{0}^{2} k^{2} \delta_{1}(k) \\
& +4 r^{7} b_{0}^{3} k^{3} \delta_{1}(k) b_{0}^{2} k^{3} \delta_{1}(k)+2 r^{7} b_{0}^{3} k^{2} \delta_{1}(k) b_{0}^{2} k^{4} \delta_{1}(k) \\
& +2 \tau_{1} r^{7} b_{0}^{3} k^{4} \delta_{1}(k) b_{0}^{2} k^{2} \delta_{2}(k)+2 \tau_{1} r^{7} b_{0}^{3} k^{4} \delta_{2}(k) b_{0}^{2} k^{2} \delta_{1}(k) \\
& +4 \tau_{1} r^{7} b_{0}^{3} k^{3} \delta_{1}(k) b_{0}^{2} k^{3} \delta_{2}(k)+4 \tau_{1} r^{7} b_{0}^{3} k^{3} \delta_{2}(k) b_{0}^{2} k^{3} \delta_{1}(k) \\
& +2 \tau_{1} r^{7} b_{0}^{3} k^{2} \delta_{1}(k) b_{0}^{2} k^{4} \delta_{2}(k)+2 \tau_{1} r^{7} b_{0}^{3} k^{2} \delta_{2}(k) b_{0}^{2} k^{4} \delta_{1}(k) \\
& +2|\tau|^{2} r^{7} b_{0}^{3} k^{4} \delta_{2}(k) b_{0}^{2} k^{2} \delta_{2}(k)+4|\tau|^{2} r^{7} b_{0}^{3} k^{3} \delta_{2}(k) b_{0}^{2} k^{3} \delta_{2}(k) \\
& +2|\tau|^{2} r^{7} b_{0}^{3} k^{2} \delta_{2}(k) b_{0}^{2} k^{4} \delta_{2}(k),
\end{aligned}
$$

can be replaced by terms with $b_{0}$ in the middle using

$$
\partial_{r}\left(b_{0}\right)=-2 r k^{2} b_{0}^{2}
$$

and integration by parts. For example

$$
\int_{0}^{\infty} r^{4} b_{0}^{2} k^{2} \delta_{1}(k) r b_{0}^{2} k^{2} \delta_{1}(k)=\frac{1}{2} \int_{0}^{\infty} \partial_{r}\left(r^{4} b_{0}^{2}\right) k^{2} \delta_{1}(k) b_{0} \delta_{1}(k) .
$$


After this replacement and combining the result with the terms listed in Section 4.2, we obtain the following terms (up to an overall factor of $\frac{2 \pi}{\tau_{2}}$ ):

$$
\begin{aligned}
T= & -2|\tau|^{2} r^{7} b_{0}^{4} k^{4} \delta_{2}(k) b_{0} k^{2} \delta_{2}(k)-2 \tau_{1} r^{7} b_{0}^{4} k^{4} \delta_{2}(k) b_{0} k^{2} \delta_{1}(k) \\
& -2 \tau_{1} r^{7} b_{0}^{4} k^{4} \delta_{1}(k) b_{0} k^{2} \delta_{2}(k)-2 r^{7} b_{0}^{4} k^{4} \delta_{1}(k) b_{0} k^{2} \delta_{1}(k) \\
& -4|\tau|^{2} r^{7} b_{0}^{4} k^{5} \delta_{2}(k) b_{0} k \delta_{2}(k)-4 \tau_{1} r^{7} b_{0}^{4} k^{5} \delta_{2}(k) b_{0} k \delta_{1}(k) \\
& -4 \tau_{1} r^{7} b_{0}^{4} k^{5} \delta_{1}(k) b_{0} k \delta_{2}(k)-4 r^{7} b_{0}^{4} k^{5} \delta_{1}(k) b_{0} k \delta_{1}(k) \\
& -2|\tau|^{2} r^{7} b_{0}^{4} k^{6} \delta_{2}(k) b_{0} \delta_{2}(k)-2 \tau_{1} r^{7} b_{0}^{4} k^{6} \delta_{2}(k) b_{0} \delta_{1}(k) \\
& -2 \tau_{1} r^{7} b_{0}^{4} k^{6} \delta_{1}(k) b_{0} \delta_{2}(k)-2 r^{7} b_{0}^{4} k^{6} \delta_{1}(k) b_{0} \delta_{1}(k) \\
& +4|\tau|^{2} r^{5} b_{0}^{3} k^{3} \delta_{2}(k) b_{0} k \delta_{2}(k)+4 \tau_{1} r^{5} b_{0}^{3} k^{3} \delta_{2}(k) b_{0} k \delta_{1}(k) \\
& +4 \tau_{1} r^{5} b_{0}^{3} k^{3} \delta_{1}(k) b_{0} k \delta_{2}(k)+4 r^{5} b_{0}^{3} k^{3} \delta_{1}(k) b_{0} k \delta_{1}(k) \\
& +4|\tau|^{2} r^{5} b_{0}^{3} k^{4} \delta_{2}(k) b_{0} \delta_{2}(k)+4 r^{5} b_{0}^{3} k^{4} \delta_{1}(k) b_{0} \delta_{1}(k) \\
& +8 \tau_{1} r^{5} b_{0}^{3} k^{4} \delta_{1}(k) b_{0} \delta_{2}(k)-2|\tau|^{2} r^{3} b_{0}^{2} k^{2} \delta_{2}(k) b_{0} \delta_{2}(k) \\
& +2 \tau_{1} r^{3} b_{0}^{2} k^{2} \delta_{2}(k) b_{0} \delta_{1}(k)-6 \tau_{1} r^{3} b_{0}^{2} k^{2} \delta_{1}(k) b_{0} \delta_{2}(k) \\
& -2 r^{3} b_{0}^{2} k^{2} \delta_{1}(k) b_{0} \delta_{1}(k) .
\end{aligned}
$$

4.4. Integrating the terms with $\boldsymbol{b}_{\mathbf{0}}$ in the middle. The computation of $\int_{0}^{\infty} \bullet d r$ of these terms is achieved by the following lemma of Connes and Tretkoff proved in [8].

Lemma 4.2. For any $\rho \in A_{\theta}^{\infty}$ and every non-negative integer $m$, one has

$$
\int_{0}^{\infty} \frac{k^{2 m+2} u^{m}}{\left(k^{2} u+1\right)^{m+1}} \rho \frac{1}{\left(k^{2} u+1\right)} d u=\mathscr{D}_{m}(\rho),
$$

where $\mathscr{D}_{m}=\mathscr{L}_{m}(\Delta), \Delta$ is the modular automorphism introduced in Section 2, and $\mathscr{L}_{m}$ is the modified logarithm

$$
\begin{aligned}
\mathscr{L}_{m}(u) & =\int_{0}^{\infty} \frac{x^{m}}{(x+1)^{m+1}} \frac{1}{(x u+1)} d x \\
& =(-1)^{m}(u-1)^{-(m+1)}\left(\log u-\sum_{j=1}^{m}(-1)^{j+1} \frac{(u-1)^{j}}{j}\right) .
\end{aligned}
$$

Now we write $T$ as a sum of three terms $T=T_{1}+T_{2}+T_{3}$ in a way that the above lemma will be applied to each $T_{j}$ by choosing $m=j$ for $j=1,2,3$. We let

$$
\begin{aligned}
T_{1}= & -2|\tau|^{2} r^{3} b_{0}^{2} k^{2} \delta_{2}(k) b_{0} \delta_{2}(k)+2 \tau_{1} r^{3} b_{0}^{2} k^{2} \delta_{2}(k) b_{0} \delta_{1}(k) \\
& -6 \tau_{1} r^{3} b_{0}^{2} k^{2} \delta_{1}(k) b_{0} \delta_{2}(k)-2 r^{3} b_{0}^{2} k^{2} \delta_{1}(k) b_{0} \delta_{1}(k) .
\end{aligned}
$$


Gauss-Bonnet theorem for noncommutative 2-tori with a general conformal structure 473

With the substitution $u=r^{2}$, we have $r d r=\frac{1}{2} d u$, and using Lemma 4.2, $\int_{0}^{\infty} \bullet d r$ of these terms (up to an overall factor of $\frac{2 \pi}{\tau_{2}}$ ) is computed respectively as follows:

$$
\begin{aligned}
& -|\tau|^{2} k^{-2} \int_{0}^{\infty} \frac{k^{4} u}{\left(k^{2} u+1\right)^{2}} \delta_{2}(k) \frac{1}{k^{2} u+1} \delta_{2}(k) d u \\
& +\tau_{1} k^{-2} \int_{0}^{\infty} \frac{k^{4} u}{\left(k^{2} u+1\right)^{2}} \delta_{2}(k) \frac{1}{k^{2} u+1} \delta_{1}(k) d u \\
& -3 \tau_{1} k^{-2} \int_{0}^{\infty} \frac{k^{4} u}{\left(k^{2} u+1\right)^{2}} \delta_{1}(k) \frac{1}{k^{2} u+1} \delta_{2}(k) d u \\
& -k^{-2} \int_{0}^{\infty} \frac{k^{4} u}{\left(k^{2} u+1\right)^{2}} \delta_{1}(k) \frac{1}{k^{2} u+1} \delta_{1}(k) d u \\
& =-|\tau|^{2} k^{-2} \mathscr{D}_{1}\left(\delta_{2}(k)\right) \delta_{2}(k)+\tau_{1} k^{-2} \mathscr{D}_{1}\left(\delta_{2}(k)\right) \delta_{1}(k) \\
& -3 \tau_{1} k^{-2} \mathscr{D}_{1}\left(\delta_{1}(k)\right) \delta_{2}(k)-k^{-2} \mathscr{D}_{1}\left(\delta_{1}(k)\right) \delta_{1}(k) .
\end{aligned}
$$

Then we let

$$
\begin{aligned}
T_{2}= & 4|\tau|^{2} r^{5} b_{0}^{3} k^{3} \delta_{2}(k) b_{0} k \delta_{2}(k)+4 \tau_{1} r^{5} b_{0}^{3} k^{3} \delta_{2}(k) b_{0} k \delta_{1}(k) \\
& +4 \tau_{1} r^{5} b_{0}^{3} k^{3} \delta_{1}(k) b_{0} k \delta_{2}(k)+4 r^{5} b_{0}^{3} k^{3} \delta_{1}(k) b_{0} k \delta_{1}(k) \\
& +4|\tau|^{2} r^{5} b_{0}^{3} k^{4} \delta_{2}(k) b_{0} \delta_{2}(k)+4 r^{5} b_{0}^{3} k^{4} \delta_{1}(k) b_{0} \delta_{1}(k) \\
& +8 \tau_{1} r^{5} b_{0}^{3} k^{4} \delta_{1}(k) b_{0} \delta_{2}(k),
\end{aligned}
$$

and compute $\int_{0}^{\infty} \bullet d r$ of these terms respectively (up to an overall factor of $\frac{2 \pi}{\tau_{2}}$ ) as follows. Using $k \delta_{j}(k) k=k^{2} \Delta^{1 / 2}\left(\delta_{j}(k)\right)$, we have

$$
\begin{aligned}
2|\tau|^{2} & \int_{0}^{\infty} \frac{k^{3} u^{2}}{\left(k^{2} u+1\right)^{3}} \delta_{2}(k) \frac{k}{k^{2} u+1} \delta_{2}(k) d u \\
& =2|\tau|^{2} k^{-2} \int_{0}^{\infty} \frac{k^{6} u^{2}}{\left(k^{2} u+1\right)^{3}} \Delta^{1 / 2}\left(\delta_{2}(k)\right) \frac{1}{k^{2} u+1} \delta_{2}(k) d u \\
& =2|\tau|^{2} k^{-2} \mathscr{D}_{2} \Delta^{1 / 2}\left(\delta_{2}(k)\right) \delta_{2}(k) ;
\end{aligned}
$$

similarly one has

$$
\begin{gathered}
2 \tau_{1} \int_{0}^{\infty} \frac{k^{3} u^{2}}{\left(k^{2} u+1\right)^{3}} \delta_{2}(k) \frac{1}{k^{2} u+1} k \delta_{1}(k) d u=2 \tau_{1} k^{-2} \mathscr{D}_{2} \Delta^{1 / 2}\left(\delta_{2}(k)\right) \delta_{1}(k), \\
2 \tau_{1} \int_{0}^{\infty} \frac{k^{3} u^{2}}{\left(k^{2} u+1\right)^{3}} \delta_{1}(k) \frac{1}{k^{2} u+1} k \delta_{2}(k) d u=2 \tau_{1} k^{-2} \mathscr{D}_{2} \Delta^{1 / 2}\left(\delta_{1}(k)\right) \delta_{2}(k), \\
2 \int_{0}^{\infty} \frac{k^{3} u^{2}}{\left(k^{2} u+1\right)^{3}} \delta_{1}(k) \frac{1}{k^{2} u+1} k \delta_{1}(k) d u=2 k^{-2} \mathscr{D}_{2} \Delta^{1 / 2}\left(\delta_{1}(k)\right) \delta_{1}(k) .
\end{gathered}
$$

Also we have

$$
2|\tau|^{2} k^{-2} \int_{0}^{\infty} \frac{k^{6} u^{2}}{\left(k^{2} u+1\right)^{3}} \delta_{2}(k) \frac{1}{k^{2} u+1} \delta_{2}(k) d u=2|\tau|^{2} k^{-2} \mathscr{D}_{2}\left(\delta_{2}(k)\right) \delta_{2}(k),
$$




$$
2 k^{-2} \int_{0}^{\infty} \frac{k^{6} u^{2}}{\left(k^{2} u+1\right)^{3}} \delta_{1}(k) \frac{1}{k^{2} u+1} \delta_{1}(k) d u=2 k^{-2} \mathscr{D}_{2}\left(\delta_{1}(k)\right) \delta_{1}(k) .
$$

In order to obtain symmetry in the result, we treat the last term of $T_{2}$ more carefully. Since

$$
\partial_{r}\left(r^{4} b_{0}^{2}\right)=4 r^{3} b_{0}^{2}-4 r^{5} k^{2} b_{0}^{3}
$$

we can write:

$$
\begin{aligned}
2 \tau_{1} \int_{0}^{\infty} & 4 r^{5} b_{0}^{3} k^{4} \delta_{1}(k) b_{0} \delta_{2}(k) d r \\
= & 2 \tau_{1} \int_{0}^{\infty}\left(4 r^{3} b_{0}^{2}-\partial_{r}\left(r^{4} b_{0}^{2}\right)\right) k^{2} \delta_{1}(k) b_{0} \delta_{2}(k) d r \\
= & 2 \tau_{1} \int_{0}^{\infty} 4 r^{3} b_{0}^{2} k^{2} \delta_{1}(k) b_{0} \delta_{2}(k) d r-2 \tau_{1} \int_{0}^{\infty} \partial_{r}\left(r^{4} b_{0}^{2}\right) k^{2} \delta_{1}(k) b_{0} \delta_{2}(k) d r \\
= & 4 \tau_{1} k^{-2} \int_{0}^{\infty} \frac{k^{4} u}{\left(k^{2} u+1\right)^{2}} \delta_{1}(k) \frac{1}{\left(k^{2} u+1\right)^{2}} \delta_{2}(k) d u \\
& +2 \tau_{1} \int_{0}^{\infty} r^{4} b_{0}^{2} k^{2} \delta_{1}(k) \partial_{r}\left(b_{0}\right) \delta_{2}(k) d r \\
= & 4 \tau_{1} k^{-2} \mathscr{D}_{1}\left(\delta_{1}(k)\right)-4 \tau_{1} \int_{0}^{\infty} r^{5} b_{0}^{2} k^{2} \delta_{1}(k) k^{2} b_{0}^{2} \delta_{2}(k) d r .
\end{aligned}
$$

Since we are working under the trace, we can first split the latter integral to two parts and then compute it as follows:

$$
\begin{aligned}
- & 4 \tau_{1} \int_{0}^{\infty} r^{5} b_{0}^{2} k^{2} \delta_{1}(k) k^{2} b_{0}^{2} \delta_{2}(k) d r \\
= & -2 \tau_{1} \int_{0}^{\infty} r^{5} b_{0}^{2} k^{2} \delta_{1}(k) k^{2} b_{0}^{2} \delta_{2}(k) d r-2 \tau_{1} \int_{0}^{\infty} r^{5} b_{0}^{2} k^{2} \delta_{2}(k) k^{2} b_{0}^{2} \delta_{1}(k) d r \\
= & -\tau_{1} \int_{0}^{\infty} \partial_{r}\left(r^{4} b_{0}^{2}\right) k^{2} \delta_{1}(k) b_{0} \delta_{2}(k) d r-\tau_{1} \int_{0}^{\infty} \partial_{r}\left(r^{4} b_{0}^{2}\right) k^{2} \delta_{2}(k) b_{0} \delta_{1}(k) d r \\
= & -\tau_{1} \int_{0}^{\infty}\left(4 r^{3} b_{0}^{2}-4 r^{5} k^{2} b_{0}^{3}\right) k^{2} \delta_{1}(k) b_{0} \delta_{2}(k) d r \\
& -\tau_{1} \int_{0}^{\infty}\left(4 r^{3} b_{0}^{2}-4 r^{5} k^{2} b_{0}^{3}\right) k^{2} \delta_{2}(k) b_{0} \delta_{1}(k) d r \\
= & -4 \tau_{1} \int_{0}^{\infty} r^{3} b_{0}^{2} k^{2} \delta_{1}(k) b_{0} \delta_{2}(k) d r+4 \tau_{1} \int_{0}^{\infty} r^{5} k^{2} b_{0}^{3} k^{2} \delta_{1}(k) b_{0} \delta_{2}(k) d r \\
& -4 \tau_{1} \int_{0}^{\infty} r^{3} b_{0}^{2} k^{2} \delta_{2}(k) b_{0} \delta_{1}(k) d r+4 \tau_{1} \int_{0}^{\infty} r^{5} k^{2} b_{0}^{3} k^{2} \delta_{2}(k) b_{0} \delta_{1}(k) d r
\end{aligned}
$$


Gauss-Bonnet theorem for noncommutative 2-tori with a general conformal structure 475

$$
\begin{aligned}
= & -2 \tau_{1} k^{-2} \int_{0}^{\infty} \frac{k^{4} u}{\left(k^{2} u+1\right)^{2}} \delta_{1}(k) \frac{1}{k^{2} u+1} \delta_{2}(k) d u \\
& +2 \tau_{1} k^{-2} \int_{0}^{\infty} \frac{k^{6} u^{2}}{\left(k^{2} u+1\right)^{3}} \delta_{1}(k) \frac{1}{k^{2} u+1} \delta_{2}(k) d u \\
& -2 \tau_{1} k^{-2} \int_{0}^{\infty} \frac{k^{4} u}{\left(k^{2} u+1\right)^{2}} \delta_{2}(k) \frac{1}{k^{2} u+1} \delta_{1}(k) d u \\
& +2 \tau_{1} k^{-2} \int_{0}^{\infty} \frac{k^{6} u^{2}}{\left(k^{2} u+1\right)^{3}} \delta_{2}(k) \frac{1}{k^{2} u+1} \delta_{1}(k) d u \\
= & -2 \tau_{1} k^{-2} \mathscr{D}_{1}\left(\delta_{1}(k)\right) \delta_{2}(k)+2 \tau_{1} k^{-2} \mathscr{D}_{2}\left(\delta_{1}(k)\right) \delta_{2}(k) \\
& -2 \tau_{1} k^{-2} \mathscr{D}_{1}\left(\delta_{2}(k)\right) \delta_{1}(k)+2 \tau_{1} k^{-2} \mathscr{D}_{2}\left(\delta_{2}(k)\right) \delta_{1}(k) .
\end{aligned}
$$

Therefore from (2) we have

$$
\begin{aligned}
8 \tau_{1} \int_{0}^{\infty} r^{5} b_{0}^{3} k^{4} \delta_{1}(k) b_{0} \delta_{2}(k) d r= & 2 \tau_{1} k^{-2} \mathscr{D}_{1}\left(\delta_{1}(k)\right) \delta_{2}(k) \\
& +2 \tau_{1} k^{-2} \mathscr{D}_{2}\left(\delta_{1}(k)\right) \delta_{2}(k) \\
& -2 \tau_{1} k^{-2} \mathscr{D}_{1}\left(\delta_{2}(k)\right) \delta_{1}(k) \\
& +2 \tau_{1} k^{-2} \mathscr{D}_{2}\left(\delta_{2}(k)\right) \delta_{1}(k) .
\end{aligned}
$$

Now we let

$$
\begin{aligned}
T_{3}= & -2|\tau|^{2} r^{7} b_{0}^{4} k^{4} \delta_{2}(k) b_{0} k^{2} \delta_{2}(k)-2 \tau_{1} r^{7} b_{0}^{4} k^{4} \delta_{2}(k) b_{0} k^{2} \delta_{1}(k) \\
& -2 \tau_{1} r^{7} b_{0}^{4} k^{4} \delta_{1}(k) b_{0} k^{2} \delta_{2}(k)-2 r^{7} b_{0}^{4} k^{4} \delta_{1}(k) b_{0} k^{2} \delta_{1}(k) \\
& -4|\tau|^{2} r^{7} b_{0}^{4} k^{5} \delta_{2}(k) b_{0} k \delta_{2}(k)-4 \tau_{1} r^{7} b_{0}^{4} k^{5} \delta_{2}(k) b_{0} k \delta_{1}(k) \\
& -4 \tau_{1} r^{7} b_{0}^{4} k^{5} \delta_{1}(k) b_{0} k \delta_{2}(k)-4 r^{7} b_{0}^{4} k^{5} \delta_{1}(k) b_{0} k \delta_{1}(k) \\
& --2|\tau|^{2} r^{7} b_{0}^{4} k^{6} \delta_{2}(k) b_{0} \delta_{2}(k)-2 \tau_{1} r^{7} b_{0}^{4} k^{6} \delta_{2}(k) b_{0} \delta_{1}(k) \\
& -2 \tau_{1} r^{7} b_{0}^{4} k^{6} \delta_{1}(k) b_{0} \delta_{2}(k)-2 r^{7} b_{0}^{4} k^{6} \delta_{1}(k) b_{0} \delta_{1}(k)
\end{aligned}
$$

and compute $\int_{0}^{\infty} \bullet d r$ of these terms respectively (up to an overall factor of $\frac{2 \pi}{\tau_{2}}$ ) as follows. Using $\delta_{j}(k) k^{2}=k^{2} \Delta\left(\delta_{j}(k)\right)$, we have

$$
\begin{aligned}
-|\tau|^{2} \int_{0}^{\infty} & \frac{k^{4} u^{3}}{\left(k^{2} u+1\right)^{4}} \delta_{2}(k) \frac{k^{2}}{k^{2} u+1} \delta_{2}(k) d u \\
& =-|\tau|^{2} k^{-2} \int_{0}^{\infty} \frac{k^{8} u^{3}}{\left(k^{2} u+1\right)^{4}} \Delta\left(\delta_{2}(k)\right) \frac{1}{k^{2} u+1} \delta_{2}(k) d u \\
& =-|\tau|^{2} k^{-2} D_{3} \Delta\left(\delta_{2}(k)\right) \delta_{2}(k) ;
\end{aligned}
$$


similarly one has

$$
\begin{gathered}
-\tau_{1} \int_{0}^{\infty} \frac{k^{4} u^{3}}{\left(k^{2} u+1\right)^{4}}\left(\delta_{2}(k)\right) \frac{1}{k^{2} u+1} k^{2} \delta_{1}(k) d u=-\tau_{1} k^{-2} \mathscr{D}_{3} \Delta\left(\delta_{2}(k)\right) \delta_{1}(k), \\
-\tau_{1} \int_{0}^{\infty} \frac{k^{4} u^{3}}{\left(k^{2} u+1\right)^{4}}\left(\delta_{1}(k)\right) \frac{1}{k^{2} u+1} k^{2} \delta_{2}(k) d u=-\tau_{1} k^{-2} \mathscr{D}_{3} \Delta\left(\delta_{1}(k)\right) \delta_{2}(k), \\
-\int_{0}^{\infty} \frac{k^{4} u^{3}}{\left(k^{2} u+1\right)^{4}}\left(\delta_{1}(k)\right) \frac{1}{k^{2} u+1} k^{2} \delta_{1}(k) d u=-k^{-2} \mathscr{D}_{3} \Delta\left(\delta_{1}(k)\right) \delta_{1}(k) .
\end{gathered}
$$

Also, using $k \delta_{j}(k) k=k^{2} \Delta^{1 / 2}\left(\delta_{j}(k)\right)$, we have

$$
\begin{aligned}
-4|\tau|^{2} & \int_{0}^{\infty} \frac{k^{5} u^{3}}{\left(k^{2} u+1\right)^{4}} \delta_{2}(k) \frac{k}{k^{2} u+1} \delta_{2}(k) d u \\
= & -2|\tau|^{2} k^{-2} \int_{0}^{\infty} \frac{k^{6} u^{3}}{\left(k^{2} u+1\right)^{4}} \Delta^{1 / 2}\left(\delta_{2}(k)\right) \frac{1}{k^{2} u+1} \delta_{2}(k) d u \\
& =-2|\tau|^{2} k^{-2} D_{3} \Delta^{1 / 2}\left(\delta_{2}(k)\right) \delta_{2}(k) ;
\end{aligned}
$$

similarly one has

$$
\begin{aligned}
-2 \tau_{1} \int_{0}^{\infty} \frac{k^{5} u^{3}}{\left(k^{2} u+1\right)^{4}} \delta_{2}(k) \frac{1}{k^{2} u+1} k \delta_{1}(k) d u & =-2 \tau_{1} k^{-2} \mathscr{D}_{3} \Delta^{1 / 2}\left(\delta_{2}(k)\right) \delta_{1}(k), \\
-2 \tau_{1} \int_{0}^{\infty} \frac{k^{5} u^{3}}{\left(k^{2} u+1\right)^{4}} \delta_{1}(k) \frac{1}{k^{2} u+1} k \delta_{2}(k) d u & =-2 \tau_{1} k^{-2} \mathscr{D}_{3} \Delta^{1 / 2}\left(\delta_{1}(k)\right) \delta_{2}(k), \\
-2 \int_{0}^{\infty} \frac{k^{5} u^{3}}{\left(k^{2} u+1\right)^{4}} \delta_{1}(k) \frac{1}{k^{2} u+1} k \delta_{1}(k) d u & =-2 k^{-2} \mathscr{D}_{3} \Delta^{1 / 2}\left(\delta_{1}(k)\right) \delta_{1}(k) .
\end{aligned}
$$

For the rest of the terms in $T_{3}$, we have

$$
\begin{aligned}
-|\tau|^{2} \int_{0}^{\infty} \frac{k^{6} u^{3}}{\left(k^{2} u+1\right)^{4}} \delta_{2}(k) \frac{1}{k^{2} u+1} k \delta_{2}(k) d u & =-|\tau|^{2} k^{-2} \mathscr{D}_{3}\left(\delta_{2}(k)\right) \delta_{2}(k), \\
-\tau_{1} \int_{0}^{\infty} \frac{k^{6} u^{3}}{\left(k^{2} u+1\right)^{4}} \delta_{2}(k) \frac{1}{k^{2} u+1} k \delta_{1}(k) d u & =-\tau_{1} k^{-2} \mathscr{D}_{3}\left(\delta_{2}(k)\right) \delta_{1}(k), \\
-\tau_{1} \int_{0}^{\infty} \frac{k^{6} u^{3}}{\left(k^{2} u+1\right)^{4}} \delta_{1}(k) \frac{1}{k^{2} u+1} k \delta_{2}(k) d u & =-\tau_{1} k^{-2} \mathscr{D}_{3}\left(\delta_{1}(k)\right) \delta_{2}(k), \\
-\int_{0}^{\infty} \frac{k^{6} u^{3}}{\left(k^{2} u+1\right)^{4}} \delta_{1}(k) \frac{1}{k^{2} u+1} k \delta_{1}(k) d u & =-k^{-2} \mathscr{D}_{3}\left(\delta_{1}(k)\right) \delta_{1}(k) .
\end{aligned}
$$




\section{Independence of $\zeta(0)$ from the Weyl factor}

The calculations of Section 4 allow us to express the value at the origin of the zeta function of the operator $\Delta^{\prime} \sim k \Delta k$ by a complex formula:

$$
\begin{aligned}
\zeta(0)+1= & \frac{-2 \pi}{\tau_{2}} \tau_{0}\left(-\frac{1}{6} k^{-1} \delta_{1}^{2}(k)-\frac{|\tau|^{2}}{6} k^{-1} \delta_{2}^{2}(k)-\frac{\tau_{1}}{3} k^{-1} \delta_{1} \delta_{2}(k)+\frac{1}{3} k^{-2} \delta_{1}(k)^{2}\right. \\
& +\frac{|\tau|^{2}}{3} k^{-2} \delta_{2}(k)^{2}+\frac{\tau_{1}}{3} k^{-2} \delta_{1}(k) \delta_{2}(k)+\frac{\tau_{1}}{3} k^{-2} \delta_{2}(k) \delta_{1}(k) \\
& -|\tau|^{2} k^{-2} \mathscr{D}_{1}\left(\delta_{2}(k)\right) \delta_{2}(k)+\tau_{1} k^{-2} \mathscr{D}_{1}\left(\delta_{2}(k)\right) \delta_{1}(k) \\
& -3 \tau_{1} k^{-2} \mathscr{D}_{1}\left(\delta_{1}(k)\right) \delta_{2}(k)-k^{-2} \mathscr{D}_{1}\left(\delta_{1}(k)\right) \delta_{1}(k) \\
& +2|\tau|^{2} k^{-2} \mathscr{D}_{2} \Delta^{1 / 2}\left(\delta_{2}(k)\right) \delta_{2}(k)+2 \tau_{1} k^{-2} \mathscr{D}_{2} \Delta^{1 / 2}\left(\delta_{2}(k)\right) \delta_{1}(k) \\
& +2 \tau_{1} k^{-2} \mathscr{D}_{2} \Delta^{1 / 2}\left(\delta_{1}(k)\right) \delta_{2}(k)+2 k^{-2} \mathscr{D}_{2} \Delta^{1 / 2}\left(\delta_{1}(k)\right) \delta_{1}(k) \\
& +2|\tau|^{2} k^{-2} \mathscr{D}_{2}\left(\delta_{2}(k)\right) \delta_{2}(k)+2 k^{-2} \mathscr{D}_{2}\left(\delta_{1}(k)\right) \delta_{1}(k) \\
& +2 \tau_{1} k^{-2} \mathscr{D}_{1}\left(\delta_{1}(k)\right) \delta_{2}(k)+2 \tau_{1} k^{-2} \mathscr{D}_{2}\left(\delta_{1}(k)\right) \delta_{2}(k) \\
& -2 \tau_{1} k^{-2} \mathscr{D}_{1}\left(\delta_{2}(k)\right) \delta_{1}(k)+2 \tau_{1} k^{-2} \mathscr{D}_{2}\left(\delta_{2}(k)\right) \delta_{1}(k) \\
& -|\tau|^{2} k^{-2} \mathscr{D}_{3} \Delta\left(\delta_{2}(k)\right) \delta_{2}(k)-\tau_{1} k^{-2} \mathscr{D}_{3} \Delta\left(\delta_{2}(k)\right) \delta_{1}(k) \\
& -\tau_{1} k^{-2} \mathscr{D}_{3} \Delta\left(\delta_{1}(k)\right) \delta_{2}(k)-k^{-2} \mathscr{D}_{3} \Delta\left(\delta_{1}(k)\right) \delta_{1}(k) \\
& -2|\tau|^{2} k^{-2} \mathscr{D}_{3} \Delta^{1 / 2}\left(\delta_{2}(k)\right) \delta_{2}(k)-2 \tau_{1} k^{-2} \mathscr{D}_{3} \Delta^{1 / 2}\left(\delta_{2}(k)\right) \delta_{1}(k) \\
& -2 \tau_{1} k^{-2} \mathscr{D}_{3} \Delta^{1 / 2}\left(\delta_{1}(k)\right) \delta_{2}(k)-2 k^{-2} \mathscr{D}_{3} \Delta^{1 / 2}\left(\delta_{1}(k)\right) \delta_{1}(k) \\
& -|\tau|^{2} k^{-2} \mathscr{D}_{3}\left(\delta_{2}(k)\right) \delta_{2}(k)-\tau_{1} k^{-2} \mathscr{D}_{3}\left(\delta_{2}(k)\right) \delta_{1}(k) \\
& \left.-\tau_{1} k^{-2} \mathscr{D}_{3}\left(\delta_{1}(k)\right) \delta_{2}(k)-k^{-2} \mathscr{D}_{3}\left(\delta_{1}(k)\right) \delta_{1}(k)\right) . \\
&
\end{aligned}
$$

Note that we have considered an overall change of sign since we set $\lambda=-1$ in our calculations in Section 4.

In the following lemma, similar to the work of Connes and Tretkoff [8], we modify (3) to prepare the ground for the proof of the main result of this paper, which relies on using functional calculus on the modular automorphism.

Lemma 5.1. For an irrational number $\theta$, let $k$ be an invertible positive element of $A_{\theta}^{\infty}$. Then the value $\zeta(0)$ of the zeta function $\zeta$ of the operator $\Delta^{\prime} \sim k \Delta k$ is given by

$$
\begin{aligned}
\zeta(0)+1= & \frac{2 \pi}{\tau_{2}} \varphi\left(f(\Delta)\left(\delta_{1}(k)\right) \delta_{1}(k)\right)+\frac{2 \pi|\tau|^{2}}{\tau_{2}} \varphi\left(f(\Delta)\left(\delta_{2}(k)\right) \delta_{2}(k)\right) \\
& +\frac{2 \pi \tau_{1}}{\tau_{2}} \varphi\left(f(\Delta)\left(\delta_{1}(k)\right) \delta_{2}(k)\right)+\frac{2 \pi \tau_{1}}{\tau_{2}} \varphi\left(f(\Delta)\left(\delta_{2}(k)\right) \delta_{1}(k)\right),
\end{aligned}
$$

where $\varphi(x)=\tau_{0}\left(x k^{-2}\right), \tau_{0}$ is the unique trace on $A_{\theta}, \Delta$ is the modular automor- 
phism, and

$$
f(u)=\frac{1}{6} u^{-1 / 2}-\frac{1}{3}+\mathscr{L}_{1}(u)-2\left(1+u^{1 / 2}\right) \mathscr{L}_{2}(u)+\left(1+u^{1 / 2}\right)^{2} \mathscr{L}_{3}(u),
$$

where for each positive integer $m, \mathscr{L}_{m}$ is the modified logarithm:

$$
\mathscr{L}_{m}(u)=(-1)^{m}(u-1)^{-(m+1)}\left(\log u-\sum_{j=1}^{m}(-1)^{j+1} \frac{(u-1)^{j}}{j}\right) .
$$

Proof. Under the trace, one can replace the first three terms in (3), namely

$$
-\frac{1}{6} k^{-1} \delta_{1}^{2}(k)-\frac{|\tau|^{2}}{6} k^{-1} \delta_{2}^{2}(k)-\frac{\tau_{1}}{3} k^{-1} \delta_{1} \delta_{2}(k)
$$

by

$$
\begin{aligned}
& -\frac{1}{6} k^{-2} \Delta^{-1 / 2}\left(\delta_{1}(k)\right) \delta_{1}(k)-\frac{|\tau|^{2}}{6} k^{-2} \Delta^{-1 / 2}\left(\delta_{2}(k)\right) \delta_{2}(k) \\
& -\frac{\tau_{1}}{6} k^{-2} \Delta^{-1 / 2}\left(\delta_{1}(k)\right) \delta_{2}(k)-\frac{\tau_{1}}{6} k^{-2} \Delta^{-1 / 2}\left(\delta_{2}(k)\right) \delta_{1}(k),
\end{aligned}
$$

because for $i, j=1,2$ one has

$$
\begin{aligned}
\tau_{0}\left(k^{-1} \delta_{i} \delta_{j}(k)\right) & =-\tau_{0}\left(\delta_{i}\left(k^{-1}\right) \delta_{j}(k)\right) \\
& =\tau_{0}\left(k^{-1} \delta_{i}(k) k^{-1} \delta_{j}(k)\right)=\tau_{0}\left(k^{-2} k \delta_{i}(k) k^{-1} \delta_{j}(k)\right) \\
& =\tau_{0}\left(k^{-2} \Delta^{-1 / 2}\left(\delta_{i}(k)\right) \delta_{j}(k)\right) .
\end{aligned}
$$

After this replacement, the terms of (3) are easily compared with the expressions in the statement of this lemma.

In the following theorem, we show that the value at the origin of the zeta function of the operator $\Delta^{\prime} \sim k \Delta k$ is independent of the choice of the invertible positive element $k \in A_{\theta}^{\infty}$ considered as the Weyl factor by which we changed the metric within its conformal class. Thus, for an irrational number $\theta$, we have proved the analogue of the Gauss-Bonnet theorem for the noncommutative two torus $A_{\theta}$ endowed with the conformal structure associated with a complex number $\tau \in \mathbb{C} \backslash \mathbb{R}$.

Theorem 5.1. For an irrational number $\theta$, let $k$ be an invertible positive element of $A_{\theta}^{\infty}$. Then the value $\zeta(0)$ of the zeta function $\zeta$ of the operator $\Delta^{\prime} \sim k \Delta k$ is independent of $k$.

Proof. The function $f(u)$ in the statement of Lemma 5.1 is of the form $h(\log u)$ where $h$ is the entire function [8]

$$
h(x)=-\frac{e^{-x / 2}\left(-1+3 e^{x / 2}+3 e^{x}+6 e^{3 x / 2} x-3 e^{2 x}-3 e^{5 x / 2}+e^{3 x}\right)}{6\left(-1+e^{x / 2}\right)^{4}\left(1+e^{x / 2}\right)^{2}} .
$$


Now, using the trace property of $\tau_{0}$, the fact that left multiplication by $k^{-1}$ commutes with any function of $\Delta$, and the following identities (cf. [8]):

$$
\begin{aligned}
k^{-1} \delta_{j}(k) & =2 \frac{\Delta^{1 / 2}-1}{\log \Delta}\left(\delta_{j}(\log k)\right), \quad j=1,2, \\
\delta_{j}(k) k^{-1} & =-2 \frac{\Delta^{-1 / 2}-1}{\log \Delta}\left(\delta_{j}(\log k)\right), \quad j=1,2, \\
\tau_{0}(a F(\log \Delta)(b)) & =\tau_{0}(F(-\log \Delta)(a) b) \quad \text { for all } a, b \in A_{\theta}^{\infty},
\end{aligned}
$$

where $F$ is an entire function. For $i, j=1,2$, we have

$$
\begin{aligned}
\varphi(f(\Delta) & \left.\left(\delta_{i}(k)\right) \delta_{j}(k)\right) \\
& =\tau_{0}\left(f(\Delta)\left(\delta_{i}(k)\right) \delta_{j}(k) k^{-2}\right) \\
& =\tau_{0}\left(f(\Delta)\left(k^{-1} \delta_{i}(k)\right) \delta_{j}(k) k^{-1}\right) \\
& =\tau_{0}\left(h(\log \Delta)\left(k^{-1} \delta_{i}(k)\right) \delta_{j}(k) k^{-1}\right) \\
& =\tau_{0}\left(h(\log \Delta)\left(2 \frac{\Delta^{1 / 2}-1}{\log \Delta}\left(\delta_{i}(\log k)\right)\right)\left(-2 \frac{\Delta^{-1 / 2}-1}{\log \Delta}\left(\delta_{j}(\log k)\right)\right)\right) \\
& =\tau_{0}\left(K(\log \Delta)\left(\delta_{i}(\log k)\right) \delta_{j}(\log k)\right),
\end{aligned}
$$

where the function $K$ (using (5)) is given by

$$
K(x)=-2 \frac{e^{x / 2}-1}{-x} h(x) 2 \frac{e^{x / 2}-1}{x}=4 x^{-2}\left(e^{x / 2}-1\right)^{2} h(x) .
$$

In fact [8],

$$
K(x)=-\frac{x-\operatorname{sh}\left[\frac{x}{2}\right]-\operatorname{sh}[x]+\frac{1}{3} \operatorname{sh}\left[\frac{3 x}{2}\right]}{x^{2} \operatorname{sh}\left[\frac{x}{2}\right]^{2}} .
$$

In particular, $K$ is an odd function. Thus, using the trace property of $\tau_{0}$ and (5), from (6) one has

$$
\begin{aligned}
\varphi\left(f(\Delta)\left(\delta_{i}(k)\right) \delta_{j}(k)\right) & =\tau_{0}\left(K(\log \Delta)\left(\delta_{i}(\log k)\right) \delta_{j}(\log k)\right) \\
& =\tau_{0}\left(\delta_{j}(\log k) K(\log \Delta)\left(\delta_{i}(\log k)\right)\right) \\
& =\tau_{0}\left(K(-\log \Delta)\left(\delta_{j}(\log k)\right) \delta_{i}(\log k)\right) \\
& =-\tau_{0}\left(K(\log \Delta)\left(\delta_{j}(\log k)\right) \delta_{i}(\log k)\right) \\
& =-\varphi\left(f(\Delta)\left(\delta_{j}(k)\right) \delta_{i}(k)\right) .
\end{aligned}
$$

Therefore $\varphi\left(f(\Delta)\left(\delta_{j}(k)\right) \delta_{j}(k)\right)=0$ for $j=1,2$ and $\varphi\left(f(\Delta)\left(\delta_{1}(k)\right) \delta_{2}(k)\right)=$ 
$-\varphi\left(f(\Delta)\left(\delta_{2}(k)\right) \delta_{1}(k)\right)$. Now the desired independence follows from (4):

$$
\begin{aligned}
\zeta(0)+1= & \frac{2 \pi}{\tau_{2}} \varphi\left(f(\Delta)\left(\delta_{1}(k)\right) \delta_{1}(k)\right)+\frac{2 \pi|\tau|^{2}}{\tau_{2}} \varphi\left(f(\Delta)\left(\delta_{2}(k)\right) \delta_{2}(k)\right) \\
& +\frac{2 \pi \tau_{1}}{\tau_{2}} \varphi\left(f(\Delta)\left(\delta_{1}(k)\right) \delta_{2}(k)\right)+\frac{2 \pi \tau_{1}}{\tau_{2}} \varphi\left(f(\Delta)\left(\delta_{2}(k)\right) \delta_{1}(k)\right) \\
= & 0 .
\end{aligned}
$$

\section{References}

[1] S. Baaj, Calcul pseudo-différentiel et produits croisés de $C^{*}$-algèbres. I et II. C. R. Acad. Sci. Paris Sér. I Math. 307 (1988), 581-586; ibid. 307 (1988), 663-666. Zbl 0655.46055 Zbl 0770.46027 MR 967366 MR 0967808

[2] A. H. Chamseddine and A. Connes, The spectral action principle. Comm. Math. Phys. 186 (1997), 731-750. Zbl 0894.58007 MR 1463819

[3] A. H. Chamseddine and A. Connes, Scale invariance in the spectral action. J. Math. Phys. 47 (2006), 063504, 19 pp. Zbl 1112.83036 MR 2239979

[4] P. B. Cohen and A. Connes, Conformal geometry of the irrational rotation algebra. Preprint MPI/92-93 (1992), 12 pp. http://www.mpim-bonn.mpg.de/preblob/4766

[5] A. Connes, $C^{*}$ algèbres et géométrie différentielle. C. R. Acad. Sci. Paris Sér. A 290 (1980), 599-604. Zbl 0433.46057 MR 572645

[6] A. Connes, Noncommutative geometry. Academic Press, San Diego, CA, 1994. Zbl 0818.46076 MR 1303779

[7] A. Connes and H. Moscovici, Type III and spectral triples. In Traces in number theory, geometry and quantum fields, Aspects Math. E38, Friedr. Vieweg, Wiesbaden 2008, 57-71. Zbl 1159.46041 MR 2427588

[8] A. Connes and P. Tretkoff, The Gauss-Bonnet theorem for the noncommutative two torus. In Noncommutative geometry, arithmetic, and related topics, Johns Hopkins University Press, Baltimore, MD, 2011, 141-158. MR 2907006

[9] P. B. Gilkey, Invariance theory, the heat equation, and the Atiyah-Singer index theorem. Mathematics Lecture Series 11, Publish or Perish Inc., Wilmington, DE, 1984. Zbl 0565.58035 MR 783634

[10] S. Rosenberg, The Laplacian on a Riemannian manifold. London Math. Soc. Stud. Texts 31, Cambridge University Press, Cambridge 1997. Zbl 0868.58074 MR 1462892

Received July 10, 2010

F. Fathizadeh, Department of Mathematics, Middlesex College, The University of Western Ontario, London, Ontario N6A 5B7, Canada

E-mail: ffathiz@uwo.ca

M. Khalkhali, Department of Mathematics, Middlesex College, The University of Western Ontario, London, Ontario N6A 5B7, Canada

E-mail:masoud@uwo.ca 